\title{
Concentration Dependence of Diffusion-Limited Reaction Rates and Its Consequences
}

\author{
Sumantra Sarkar@ \\ Center for Nonlinear Studies, Theoretical Division, Los Alamos National Laboratory, \\ Los Alamos, New Mexico 87545, USA
}

(Received 17 June 2020; revised 2 September 2020; accepted 2 October 2020; published 13 November 2020)

\begin{abstract}
Diffusion-limited association reactions are ubiquitous in nature. They are particularly important for biological reactions, where the reaction rates are often determined by the diffusive transport of the molecules on two-dimensional surfaces, such as the cell membrane. The peculiarities of diffusion on twodimensional surfaces may lead to nontrivial reaction kinetics, such as a concentration-dependent rate of association between two molecules. However, traditionally, the kinetics of biomolecular association reactions has been modeled using the law of mass action, which assumes that the rate of reaction is a concentration-independent constant. In this paper, using multiscale molecular simulation, we investigate the concentration dependence of diffusion-limited association reactions on 2D surfaces. In particular, we quantify the influence of short-ranged pair interactions on the concentration dependence of the reaction rates and codify it in an empirical law. Using this law in a chemical kinetic model, we find that the steadystate behaviors of simple chemical systems are drastically modified by the presence of concentrationdependent rates. In particular, we find that it leads to suppression of intrinsic noise in dimerization reaction and destabilizes robust oscillation in Lotka-Volterra predator-prey systems. In fact, we see a transition from robust to fine-tuned behavior in the steady-state oscillations. In addition, we show that concentrationdependent reaction rates arise naturally in stochastic predator-prey systems due to intrinsic noise. We comment on the consequences of these results and discuss their implications in the modeling of complex chemical and biological systems. In particular, we comment on the range of validity of the law of mass action, which is a staple in all theoretical modeling of these systems.
\end{abstract}

DOI: $10.1103 /$ PhysRevX.10.041032

\section{INTRODUCTION}

Association reactions are a type of elementary reaction, in which two or more reactant molecules form one or more product molecules. Formation of a dimer from two monomers is an example of an association reaction, as is the formation of water from hydrogen and oxygen [1]. Because of its elementary nature, association reactions play a central role in many physicochemical processes, including pattern formation [2,3], aggregation [4], and cell signaling [5-9]. A typical association reaction involves two steps [10-13]. In the first step, two molecules are transported near each other through some transport processes. Once the two molecules encounter each other, in the second step, the molecules interact with each other with an intrinsic association rate $\kappa_{I}$ to form the product molecule. It is usually assumed that the second step is much slower compared to the first step, such

Published by the American Physical Society under the terms of the Creative Commons Attribution 4.0 International license. Further distribution of this work must maintain attribution to the author(s) and the published article's title, journal citation, and DOI.
Subject Areas: Biological Physics, Chemical Physics, Nonlinear Dynamics

that an association reaction occurs after many encounter events. These assumptions have two repercussions: (a) One can assume that the reactant molecules are well mixed, so that the reaction rate is solely determined by the interaction step, and (b) the formation of the product molecule follows a Poisson process, such that the rate of association reaction is a time- and concentration-independent constant [14]. When combined, these two observations lead to the celebrated law of mass action (LMA) $[1,15]$, which states that the propensity of an association reaction is equal to the product of the constant reaction rate $k_{0}$ and the mass action $\Phi$, where the latter is the total number of possible reactant pairs. In particular, if we consider the following association reaction

$$
A+B \stackrel{k_{0}}{\longrightarrow} A B
$$

then the LMA states that the propensity $r$ of the association reaction is

$$
r=k_{0}[A][B]=k_{0} \Phi
$$


where [.] denotes the concentration of a molecule, and $\Phi=$ $[A][B]$ is the mass action.

The LMA is strictly valid when the rate $k_{0}$ is a concentration-independent constant. For example, the LMA works well for dilute solutions, where this "rate law" was originally developed. However, its range of applicability did not stay confined within just the purview of dilute solutions. Its simplicity and the ubiquity of association reactions have led to its application in disparate problems [16] with varying amount of success. However, it is unclear whether some of the assumptions underlying the LMA are still valid for these systems. For example, most cell-signaling reactions occur on two-dimensional cell membranes, where, often, the intrinsic reaction rates are higher than or comparable to the diffusive encounter rate, such that the reaction kinetics depends crucially on the diffusive transport of the molecules. In particular, the peculiarities of diffusion in two dimensions (2D), including nonzero probability of an encounter between all particles $[17,18]$, can result in concentration-dependent diffusive encounter rates, which, in turn, results in a concentrationdependent $k_{0}$, which invalidates the application of the LMA in such situations [19,20]. Furthermore, a fundamental assumption of the LMA is that the molecules are essentially point particles that interact with each other only when they collide with each other [21]. In reality, most molecules interact with each other through finite, albeit short-ranged, interactions, which may also influence the diffusion-limited reaction rates. In fact, in such situations also, the diffusionlimited reaction rate $\kappa$ may become concentration dependent $[14,19,22]$ and violate the assumptions of the LMA.

A practical work-around to this challenge is to use an adaptive, concentration-dependent, rate of association that varies with time $[10,17,18,23,24]$. Use of such adaptive reaction rates in chemical kinetic models drastically improves the prediction of the transient kinetics. Unfortunately, the functional form of the adaptive rates used in these studies is complex and not immediately conducive to analytical treatments. Hence, in this paper, we offer a complementary empirical formulation of the concentration dependence, which allows us to do analytical computations of the steady-state properties. The feasibility of the analytical treatment offers practical advantages to explore the consequences of the concentration dependence for system parameters that are difficult to explore through simulation.

To theoretically study the concentration dependence of the diffusion-limited reaction rates and to construct an empirical law at concentrations relevant to most applications, one has to simulate the transport and interaction of the molecules in large spatially heterogeneous systems. The main challenge to such a line of enquiry is that diffusion is difficult to investigate through molecular simulation. In this paper, we overcome this challenge by using a recently developed multiscale simulation framework called the
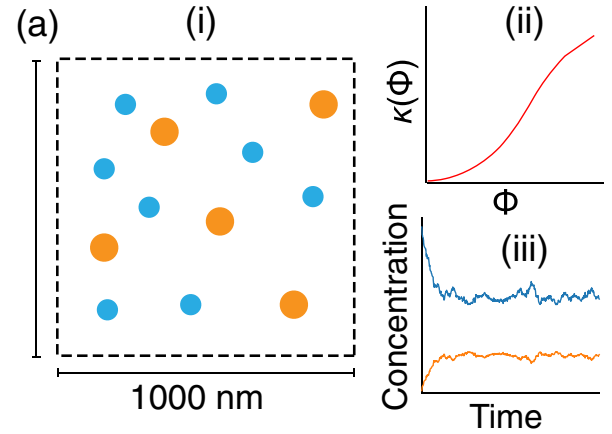

(b)
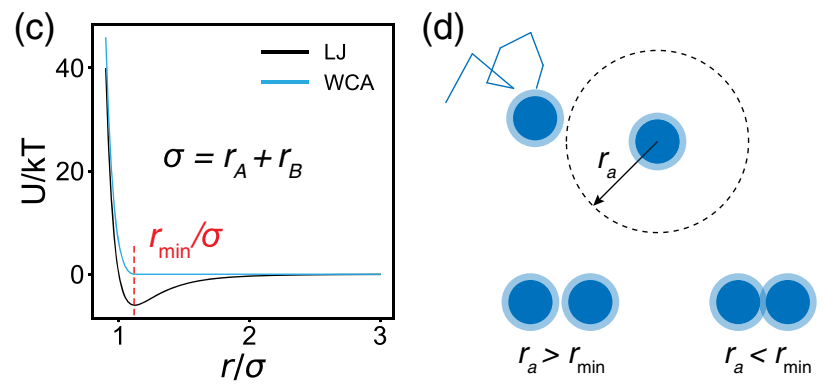

FIG. 1. Hierarchical multiscale simulation framework: (A) We combine molecular simulation with the chemical kinetic model to study association reaction kinetics at broad spatiotemporal scales. (i) Using BD-GFRD, we study how short-ranged pairwise interaction modifies the rate of association reaction between two molecules on a $2 \mathrm{D}$ periodic box of size $1 \times 1 \mu \mathrm{m}^{2}$. (ii) The measured rate $\kappa$ is concentration dependent, and its dependence on concentration is represented by the function $\kappa(\Phi)$, where $\Phi$ is the product of the concentrations of the reactants, aka the mass action. (iii) $\kappa(\Phi)$ is used in a spatially homogeneous chemical kinetic model to study concentration variations at timescales not reachable through molecular simulations ( $>1 \mathrm{~s})$. (B) The molecules are represented as disks of radius $r_{A}$ and $r_{B}$, and (c) they interact through either Lennard-Jones (LJ) or Weeks-ChandlerAndersen (WCA) interaction. (D) The molecules react as soon as they are closer than the reaction radius $r_{a}$. If $r_{a}<r_{\min }$ [see (C) and (D) for definition of $r_{\mathrm{min}}$ ], there is a strong excluded volume interaction between the reactant molecules. The light blue region surrounding a molecule denotes the region over which excluded volume interaction is felt by another molecule. More precisely, when two light blue regions touch, the distance between the two molecules is $r_{\min }$.

Green's function reaction dynamics with Brownian dynamics, or BD-GFRD [13,25-28]. We combine BD-GFRD with chemical kinetic models to construct a hierarchical multiscale simulation framework [Eq. (3)]. In the first level of hierarchy of this framework using BD-GFRD, we measure the concentration dependence of the rates and codify it in a functional form $\kappa(\Phi)$ [Figs. 1(ai) and 1(aii)]. In the next level, we use $\kappa(\Phi)$ in a chemical kinetic model to study the behavior of the chemical systems at timescales that are not reachable through BD-GFRD [Fig. 1(aiii)]. We ask, can we still use LMA even when the reaction rates are concentration dependent? In the two model systems that we study here, we find that steady-state properties obtained 
from concentration-dependent rates are qualitatively different from the LMA, but under some special conditions, this difference is negligible. Because chemical kinetic models are a staple in many lines of scientific enquiry [29], our results may provide useful guidelines and design principles for these models.

Molecular simulation $\stackrel{\text { generates }}{\longrightarrow} \kappa(\Phi) \stackrel{\text { is used to construct }}{\longrightarrow}$ chemical kinetic model $\stackrel{\text { is used to compute }}{\longrightarrow}$ steady-state behavior.

\section{CONCENTRATION DEPENDENCE OF THE DIFFUSIVE ENCOUNTER RATE}

\section{A. Molecular simulation of dimerization reactions}

To study how short-ranged pairwise interaction leads to concentration-dependent reaction rates, we use a hierarchical simulation framework, which interfaces a recently developed multiscale molecular simulation method called BD-GFRD [13,25-28] with chemical kinetic models to study association reaction kinetics at wide spatiotemporal scales and broad concentration levels [see Figs. 1(a)-1(d) and Eq. (3)]. Please see the Appendix A for a detailed description of the methods.

To measure the impact of short-ranged interactions [Fig. 1(c)] on diffusion-limited reaction rates, we investigate the concentration dependence of the rates of the following reactions:

$$
\begin{aligned}
& A+A \rightarrow A_{2}, \\
& A+B \rightarrow A B .
\end{aligned}
$$

We measure the diffusive encounter rates of these reactions in an ensemble in which the number of each species of molecules is conserved. Using this setup in the BD-GFRD [13,25-28] simulations (see Appendix A), we measure the reaction rates by varying the concentration of the monomers in the range of $2-300 / \mu \mathrm{m}^{2}$. These concentrations are similar to the concentration of integral and peripheral membrane proteins on cell membranes.

We measure the probability distribution $\psi_{D}\left(t_{D}\right)$ of the time interval $t_{D}$ between two consecutive homodimerization reactions. It has three distinct regimes separated by two timescales: the rebinding timescale $\tau_{\text {rebind }}$ and the timescale above which the reaction events are described by an exponential decay $\tau_{\text {bulk }}$ [Fig. 2(a)]. For $t_{D}<\tau_{\text {rebind, }}$, $\psi_{D}\left(t_{D}\right)$ decays as $t_{D}^{-1}$. Such a dependence occurs due to rapid rebinding of the monomers to form dimers [13]. For $\tau_{\text {rebind }}<t_{D}<\tau_{\text {bulk }}, \psi_{D}\left(t_{D}\right)$ decays as $t_{D}^{-0.2}$. We find that this region is present in all the monomer concentrations considered here [Fig. 2(b)]. As the concentration of the monomers decreases, the width of this region also decreases, which suggests that this unusual scaling results from reactions that occur before the particles have lost their memory of the previous encounter. Beyond this observation, we do not understand this scaling well and plan to investigate it in a future paper. For timescales above $\tau_{\text {bulk }}$, $\psi_{D}\left(t_{D}\right)$ decays exponentially [Fig. 2(a)]. In particular, we find that rescaling the time by $\tau_{\text {bulk }}$ collapses all distributions onto a single master curve [Fig. 2(c)], which implies that the propensity of the reactions $r$ is inversely proportional to $\tau_{\text {bulk }}$. $\tau_{\text {bulk }}$ depends on the concentration of the monomers (Fig. S1 in the Supplemental Material [30]) and at low $\Phi$ is given by
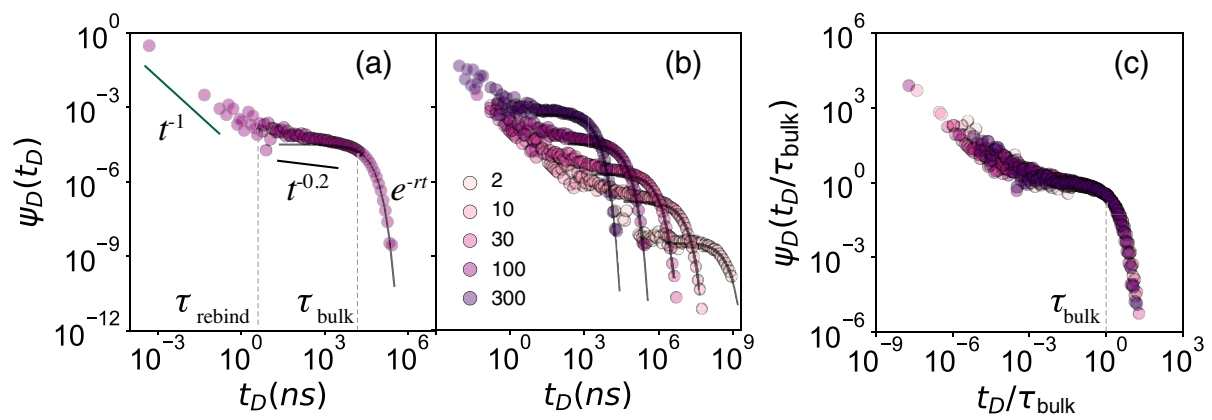

FIG. 2. (a) The dimerization time-interval distribution, $\psi_{D}\left(t_{D}\right)$. There are three distinct regions separated by two timescales $\tau_{\text {rebind }}$ and $\tau_{\text {bulk }}$. Reactions occurring at intervals $t_{D}>\tau_{\text {bulk }}$ are described by a single rate, and their propensities can be computed by fitting the exponential tail. The $t^{-1}$ distribution for $t_{D}<\tau_{\text {rebind }}$ arises because the dimerization events are time correlated due to rebinding events. (b) $\psi_{D}\left(t_{D}\right)$ for different monomer concentrations (shown in the legend with unit $\mu \mathrm{m}^{-2}$ ). The size of the region between $\tau_{\text {rebind }}<t_{D}<\tau_{\text {bulk }}$, i.e., the region with $t^{-0.2}$ scaling, decreases with decreasing monomer concentrations. In fact, at monomer concentration of $10 \mu \mathrm{m}^{-2}$, this region is almost nonexistent, as illustrated by the exponential fit. The origin of the $t^{-0.2}$ scaling remains unclear (see text). (c) Rescaling $t_{D}$ by $\tau_{\text {bulk }}$ collapses $\psi_{D}\left(t_{D}\right)$ for different concentrations onto a single master curve. 


$$
\tau_{\text {bulk }}=\frac{\log (L / a)}{8 \pi D_{A} \Phi}
$$

where $L=1000 \mathrm{~nm}$ is the system size, $a=2 r_{A}, D_{A}=$ $1 \mu \mathrm{m}^{2} \mathrm{~s}^{-1}$ is the diffusion constant of $A$, and $\Phi=[A]([A]-$ 1)/2 [18]. We observe the same behavior for the heterodimerization reactions as well (not shown). Crucially, we observe identical $\psi_{D}\left(t_{D}\right)$ in a set of simulations in which $[A]+2\left[A_{2}\right]$ is kept fixed, but concentrations of $[A]$ and $\left[A_{2}\right]$ could vary, which consolidates our observation (see Fig. S2 in the Supplemental Material [30]). One should note that, although $\psi_{D}\left(t_{D}\right)$ is described by an exponential decay in this regime, it does not imply that this process is Markovian. In 2D diffusion, due to the finite probability of reencounter between two reactants, there is not a single well-defined rate of reaction for all concentrations. Instead, the rate depends on the concentrations of the reactants, which can be used in a manner similar to the Markovian rate constant $[10,17,18,23,24]$.

\section{B. Diffusion-limited bulk dimerization rate}

The propensities $r$ of the dimerization reactions separated by $t_{D}>\tau_{\text {bulk }}$ can be computed by fitting the tail of $\psi_{D}\left(t_{D}\right)$ with an exponential function, from which we can determine $\kappa(\Phi)$, the "bulk" concentration-dependent diffusion-limited reaction rate, using the following formula:

$$
r=\kappa(\Phi) \Phi .
$$

For the LMA to hold, $\kappa(\Phi)$ should be independent of $\Phi$. However, we find that $\kappa$ varies with $\Phi$ for all pair interactions considered here [Fig. 3(a)]. Irrespective of the type of interaction potential, the functional form of $\kappa(\Phi)$ is nearly identical for $A_{2}$, except its value near $\Phi=0$. The heterodimerization reaction, i.e., the formation of $A B$, also has similar concentration dependence. However, $\kappa(\Phi)$ varies weakly compared to $A_{2}$. Finally, $\kappa(\Phi)$ also depends on the reaction radius $r_{a}[31,32]$ [Fig. 1(d)]. We find that the lower the $r_{a}$, the more slowly $\kappa(\Phi)$ increases with $\Phi$. In fact, when $r_{a}=0.89 r_{\min } \approx \sigma$, $\kappa(\Phi)$ does not change appreciably for the $\Phi$ values considered in our simulations [Fig. 3(b)]. In fact, for all the parameters considered here, as $\Phi \rightarrow 0, \kappa(\Phi)$ converges to a concentration-independent value $\kappa_{0}$, which is consistent with previous works $[14,18,33,34]$.

\section{Empirical rate law}

The preceding discussion implies that, in general, $\kappa(\Phi)$ can be expressed in terms of $\kappa_{0}$, the value of $\kappa(\Phi)$ as $\Phi \rightarrow 0$, and a function of the mass action $\Phi$. The function can be obtained by measuring the variation of $\kappa(\Phi)-\kappa_{0}$ with $\Phi$. Remarkably, we find that $\kappa(\Phi)-\kappa_{0}$ displays universal variation across different reactions studied here. In particular, for the concentrations of the monomers
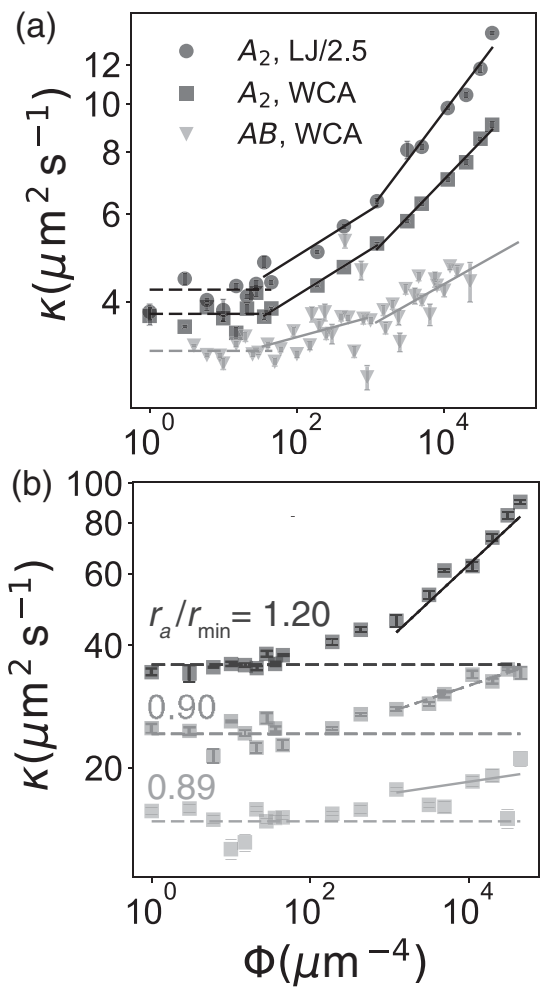

FIG. 3. Concentration-dependent diffusion-limited reaction rate: (a) The rates of association reaction $\kappa$ depend on the mass action $\Phi$. The functional form of $\kappa(\Phi)$ is independent of the interaction potential but depends on the association reaction. The solid lines are guides for the eye, whereas the dashed lines show the value of $\kappa_{0}=\lim _{\Phi \rightarrow 0} \kappa(\Phi)$. (b) $\kappa(\Phi)$ depends on the reaction radius $r_{a}$. The lower the $r_{a}$, the more slowly $\kappa(\Phi)$ increases with $\Phi$. To compute these results, we use $D=10 \mu \mathrm{m}^{2} / \mathrm{s}$. The change in diffusion constant does not change the result qualitatively (see Fig. S3 in the Supplemental Material [30]).

studied here, we find that $\kappa(\Phi)-\kappa_{0} \sim \Phi^{\epsilon}$, such that $\kappa(\Phi) \approx$ $\kappa_{0}+\kappa_{1} \Phi^{\epsilon}$, where $\epsilon=3 / 8=0.375$ [Fig. 4(a)]. Furthermore, we find that there is a mass-action value $\Phi_{0}$ such that $\kappa_{1} \Phi_{0}^{\epsilon}=\kappa_{0}$; i.e., $\Phi_{0}$ measures how quickly a reaction deviates from the concentration-independent behavior. The larger the value of $\Phi_{0}$, the slower the deviation is. Combining the above definitions, $\kappa(\Phi)$ can be succinctly written as

$$
\kappa(\Phi) \approx \kappa_{0}\left(1+\left(\frac{\Phi}{\Phi_{0}}\right)^{\epsilon}\right) .
$$

This form of $\kappa(\Phi)$ is valid for very low packing fractions of the reactants. For example, the packing fraction of the molecules at $300 / \mu \mathrm{m}^{2}$, the highest concentration of molecules studied here, is approximately $0.3 \%$, which is much lower than the packing fraction of molecules in a cellular environment (up to $40 \%$ [20]). Therefore, we expect that this approximate form of $\kappa(\Phi)$ will not hold at such high packing fractions. Instead, because of the increase in 

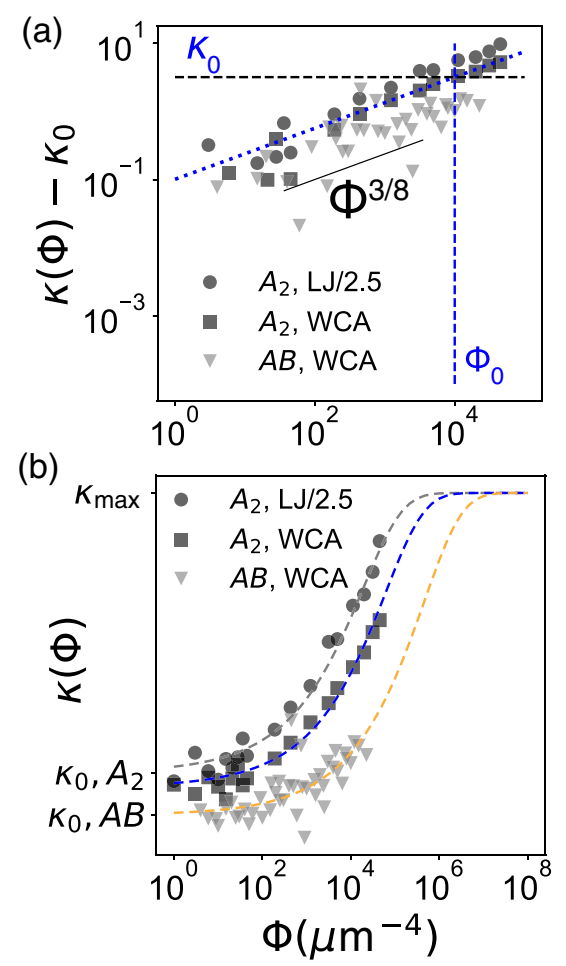

FIG. 4. Empirical rate law: (a) $\kappa(\Phi)-\kappa_{0}$ increases as $\kappa_{1} \Phi^{\epsilon}$, with $\epsilon=3 / 8$, for the $\Phi$ values considered in our simulations (blue dotted line). $\kappa_{0}$ is the concentration-independent rate, and $\Phi_{0}$ is the mass-action flux where $\kappa_{1} \Phi_{0}^{3 / 8}=\kappa_{0}$. (b) This scaling is used to postulate a sigmoidal $\kappa(\Phi)$. The parameters of the sigmoidal functions are $\kappa_{0}, \kappa_{\max }$, and $\Phi_{0}$ (see text for details). We use the postulated sigmoidal function as an empirical rate law for $\Phi$ values both encountered and not encountered in the molecular simulation. To fit these curves, we use $\kappa_{0}=$ $4.2 \mu \mathrm{m}^{2} \mathrm{~s}^{-1}$ and $\Phi_{0}=5 \times 10^{3}$ for $A_{2}$ in the presence of $\mathrm{LJ}$ interaction (dashed gray); $\kappa_{0}=3.7 \mu \mathrm{m}^{2} \mathrm{~s}^{-1}$ and $\Phi_{0}=1 \times 10^{4}$ for $A_{2}$ in the presence of WCA interaction (dashed blue); $\kappa_{0}=$ $3.2 \mu \mathrm{m}^{2} \mathrm{~s}^{-1}$ and $\Phi_{0}=1 \times 10^{5}$ for $A B$ in the presence of WCA interactions (dashed orange). The values of $\kappa_{0}$ are obtained by averaging $\kappa(\Phi)$ values for $\Phi<30$ for $D=1 \mu \mathrm{m}^{2} / \mathrm{s}$ and $r_{a}=$ $1.2 r_{\min }$ [Fig. 3(a)].

viscosity observed at high packing fractions, we expect the diffusive encounter rate $\kappa(\Phi)$ to saturate to a maximum value, $\kappa_{\max }$ [35]. It is possible to obtain the value of $\kappa_{\max }$ by doing direct simulation of the molecules at high density. Although we plan to study such a system, we do not attempt to do it in this paper. Instead, we use Smoluchowski's theory of diffusion-limited reactions [18] to obtain $\kappa_{\max }$, which for the parameters of our system is approximately $20 \mu \mathrm{m}^{2} / \mathrm{s}$ (see Appendix D). Furthermore, because $\kappa(\Phi)$ is a convex function for small $\Phi$ and saturates to a maximum value at higher $\Phi$ values, we expect its most general form to be sigmoidal. Therefore, we postulate the following empirical law that describes $\kappa(\Phi)$ for concentrations beyond the values simulated in this paper:

$$
\kappa(\Phi)=\frac{\kappa_{0} \kappa_{\max }}{\kappa_{0}+\left(\kappa_{\max }-\kappa_{0}\right) \exp \left[-\left(\frac{\Phi}{\Phi_{0}}\right)^{\epsilon}\right]} .
$$

It is easy to check that this sigmoidal $\kappa(\Phi)$ has the desired behavior for different limits of $\Phi$. Also, it fits the observed rates well [Fig. 4(b)]. In fact, the functional form that we obtain here is very similar to what has been described earlier [18]. The advantage of our approach is that the concentration dependence of the encounter rate is entirely codified by the flux $\Phi$, whereas everything else is concentration-independent parameters, and can, in principle, be obtained by using self-consistent theories of association reaction kinetics $[10,17,18,23,24]$. Empirical law in this simple form is not only useful as an input to the numerical methods, such as the Gillespie algorithm, it is also conducive to analytical treatments, such as the calculation of the steady-state concentrations and their stabilities, as we describe in the next section.

In the rest of this paper, we study the consequences of this sigmoidal rate law using chemical kinetic models (see Appendix A). For the sake of brevity, we refer to this rate law as the "law of concentration-dependent rates" or LCDR, à la the LMA. We study two model chemical systems using both the LMA and LCDR and compare the steady-state behavior of these models under the two different rate laws. We should point out that ours is by no means the first such attempt at using a chemical kinetic model to study the long-term behavior of chemical systems. In fact, there have been several papers that have pointed out that chemical kinetic equations are not accurate at low concentrations $[18,24]$. However, despite this limitation, the chemical kinetic models with concentration-dependent rate constants have been shown to reproduce kinetics of association reactions with nearly exact transient kinetics $[18,24]$. Therefore, we are justified in using such an approach to study the consequences of the concentrationdependent rates.

\section{CONSEQUENCES OF CONCENTRATION- DEPENDENT RATES}

\section{A. Suppression of intrinsic noise}

We study the consequences of the concentrationdependent reaction rates on the simplest association reaction possible: the homodimerization reaction,

$$
A+A \underset{k_{\text {off }}}{\stackrel{k_{\text {on }}}{\rightleftharpoons}} A_{2}
$$

where $k_{\mathrm{on}}=\kappa(\Phi)$ is the association rate, $\kappa_{\max }=20 \mu \mathrm{m}^{2} \mathrm{~s}^{-1}$, and $k_{\text {off }}$ is the dissociation rate of the dimer.

We study the steady-state properties of this reaction using a chemical kinetic model. To do so, we measure the 

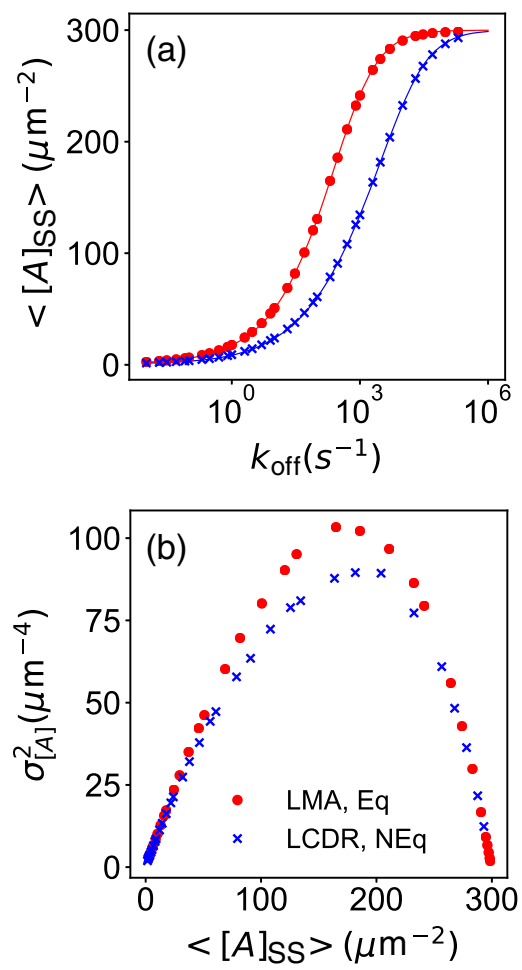

FIG. 5. Suppression of noise in dimerization reaction: (a) The mean steady-state concentration of the monomers $\left\langle[A]_{\mathrm{SS}}\right\rangle$ as a function of the dissociation rate of the dimer $k_{\text {off }}$. To compare the LMA with LCDR, we assume that $k_{\text {on }}=\kappa_{0}=4 \mu \mathrm{m}^{2} \mathrm{~s}^{-1}$ for the LMA because then $k_{\text {off }}$ is identical in both equilibrium and nonequilibrium simulations. For the LCDR, we use $\kappa_{0}=4 \mu \mathrm{m}^{2} \mathrm{~s}^{-1}, \quad \kappa_{\max }=20 \mu \mathrm{m}^{2} \mathrm{~s}^{-1}$, and $\Phi_{0}=5 \times 10^{3} \mu \mathrm{m}^{-4}$. The simulation results [see legend in (b)] match exactly with the analytically calculated values (lines). (b) The variance of monomer concentrations, $\sigma_{[A]}^{2}$ vs $\left.\langle A]_{\mathrm{SS}}\right\rangle$. The variance is identical between the LMA and LCDR at chemical equilibrium (LCDR not shown). However, in nonequilibrium steady states, the variance is lower for the LCDR than for the LMA for the same $\left\langle[A]_{\mathrm{SS}}\right\rangle$ at intermediate concentrations. For both simulations, we use $[A]+2\left[A_{2}\right]=300 \mu \mathrm{m}^{-2}$.

propensities of the association reactions using Eq. (7), where $\Phi=[A]([A]-1) / 2$ is the mass action and $\kappa(\Phi)$ is given by Eq. (9). We study the steady-state properties of this system by varying the dissociation rate $k_{\text {off }}$. To obtain equilibrium steady states, the rates have to obey detailed balance; i.e., $k_{\text {off }}$ has to be equal to $k_{\text {on }} k_{D}$, where $k_{D}$ is the equilibrium constant. To compute the mean equilibrium concentration and fluctuation at different $k_{D}$ values, we simulate the dimerization reactions using the Gillespie algorithm. The mean equilibrium concentrations $\left\langle[A]_{S S}\right\rangle$ and the variance of the fluctuations in the steady state $\sigma_{[A]}^{2}$ are identical between the LMA and LCDR [Figs. 5(a) and 5(b)], which is what we expect. To verify the correctness of the simulated results, we also calculate the steadystate concentrations analytically for both the LMA and LCDR (see Appendix B). We find that the mean monomer concentration $\left\langle[A]_{\mathrm{SS}}\right\rangle$ obtained from analytical calculation and simulation are identical [Fig. 5(a)].

To study the same quantities in a nonequilibrium steady state, we use $k_{\text {off }}=k_{D}$. For the LMA, for which $\kappa(\Phi)$ is a concentration-independent constant, this choice of the dissociation rate does not break detailed balance, and we reproduce the equilibrium mean concentration and fluctuation profile. In contrast, for the LCDR, this functional form of $k_{\text {off }}$ is sufficient to break detailed balance and obtain a nonequilibrium steady state. In addition, we discover that for same steady-state concentrations, the variance of the steady-state fluctuations are smaller in the nonequilibrium steady state of the LCDR than in the equilibrium state under both the LMA and LCDR kinetics [Fig. 5(b)]. This is a remarkable result and can be explained through a simple analogy. To do so, we first write $\kappa(\Phi)$ in its approximate form for $\Phi$ near $\Phi_{0}$ :

$$
\kappa(\Phi) \approx \kappa_{0}+\kappa_{1} \Phi^{\epsilon} .
$$

Written in this form, $r=\kappa(\Phi) \Phi$ can be interpreted as a cost function, which computes the cost associated with maintaining the system at a particular mass action value $\Phi$. The LMA, for which $\kappa_{1}$ is zero, imposes a penalty $\kappa_{0} \Delta \Phi$ for a concentration fluctuation that changes $\Phi$ to $\Phi+\Delta \Phi$. On the other hand, the LCDR imposes an additional penalty of $(1+\epsilon) \kappa_{1} \Delta \Phi \Phi^{\epsilon}$. In equilibrium, this additional cost is balanced by the reverse reaction, whose propensity is equal to $\kappa(\Phi) k_{D}\left[A_{2}\right]$. Hence, the fluctuation profiles are identical between the LMA and LCDR in equilibrium. In contrast, in the nonequilibrium state, at a given value of $\Phi$, the reverse reaction imposes a constant cost given by $k_{D}\left[A_{2}\right]$. Therefore, for all $\Phi>0$, fluctuations cost more in the LCDR than in the LMA. In fact, for the LCDR, the cost of fluctuation increases with increasing $\Phi$, but, for the LMA, the cost of fluctuation is independent of $\Phi$. That is, the difference in variance should increase with increasing monomer concentration, which is what we observe in Fig. 5 until $\left\langle[A]_{\mathrm{SS}}\right\rangle \approx 150 \mu \mathrm{m}^{-2}$, beyond which the finiteness of the system becomes important.

We must stress that the attainment of the nonequilibrium steady state is possible due to the concentration dependence of the reaction rates (see Appendix B). As we show, in the absence of the concentration dependence, as in the LMA, the system always reaches chemical equilibrium, unless the reverse reaction is time dependent. However, the reader should note that our study of the effect of the concentrationdependent reaction rate on the dimerization reaction is a toy model through which we decide to expose the impact of the concentration-dependent rate. To assess the practical relevance of our results, we still need to verify whether, in real systems, detailed balance is broken in the manner described in this paper. However, as the preceding analogy shows, the underlying reason for the noise suppression is the additional cost of fluctuation due to the concentration 
dependence, which does not depend on the choice of the nonequilibrium steady state. Therefore, the suppression of intrinsic noise by concentration-dependent rates may be a useful strategy for controlling noise.

Control of noise is an important aspect of many biological functions [36-41]. It is possible that cellular systems may control noise by modulating the local concentration of reactants on 2D surfaces, such as membranes, which can increase or decrease the amount of noise. Indeed, liquid-liquid phase separation of signaling proteins has been postulated to reduce noise in the processing of the signals $[42,43]$. Therefore, we anticipate our results will provide important insights into deciphering the nature of noise control through such biological mechanisms.

\section{B. Chemical oscillation becomes fine-tuned}

\section{Lotka-Volterra model in the presence of concentration- dependent rates}

Next, we consider the consequences of the pairwise interaction on the behavior of chemical oscillators. We study the Lotka-Volterra (LV) predator-prey equations [16], which describe the dynamics of the following set of reactions:

$$
\begin{aligned}
X & \rightarrow 2 X, \\
X+Y & \rightarrow 2 Y, \\
Y & \rightarrow \phi,
\end{aligned}
$$

where $X$ is the prey, $Y$ is the predator, and $\phi$ represents death. The most general LV equation can be written in the following way:

$$
\begin{aligned}
& \frac{d x}{d t}=\alpha x-\beta(x, y) x y, \\
& \frac{d y}{d t}=\beta(x, y) x y-\gamma y,
\end{aligned}
$$

which admits two steady-state solutions $x=y=0$ and $x=[\gamma / \beta(x, y)], y=[\alpha / \beta(x, y)]$, where $x$ and $y$ are the concentrations of $X$ and $Y$, and $\beta(x, y)=\beta(x y)$ is the concentration-dependent predation rate. We assume that $\beta(x y)$ has the same functional form as $\kappa(\Phi)$ with $\Phi=x y$ and $\Phi_{0}=10^{5} \mu \mathrm{m}^{-4}$. The concentration of the nonzero steady state depends on the kinetics used. For the LMA, i.e., $\beta(x, y)=\beta_{0}$, the steady-state concentrations increase linearly with $\alpha$ and $\gamma$. In contrast, for the LCDR, the steadystate concentrations are highly nonlinear when either $\alpha$ or $\gamma$ is large, but linear behavior is restored when both $\alpha$ and $\gamma$ are small or when the steady-state mass action $\Phi_{\mathrm{SS}} \gg \Phi_{0}$ [Fig. 6(a)].

While the $x=y=0$ steady state is a saddle point for both kinetics, the stability of the nonzero steady state depends on the underlying kinetics. For the LMA, i.e., $\beta(x, y)=\beta_{0}$, the steady state is oscillatory with frequency $\sqrt{\alpha \gamma}$ for all values
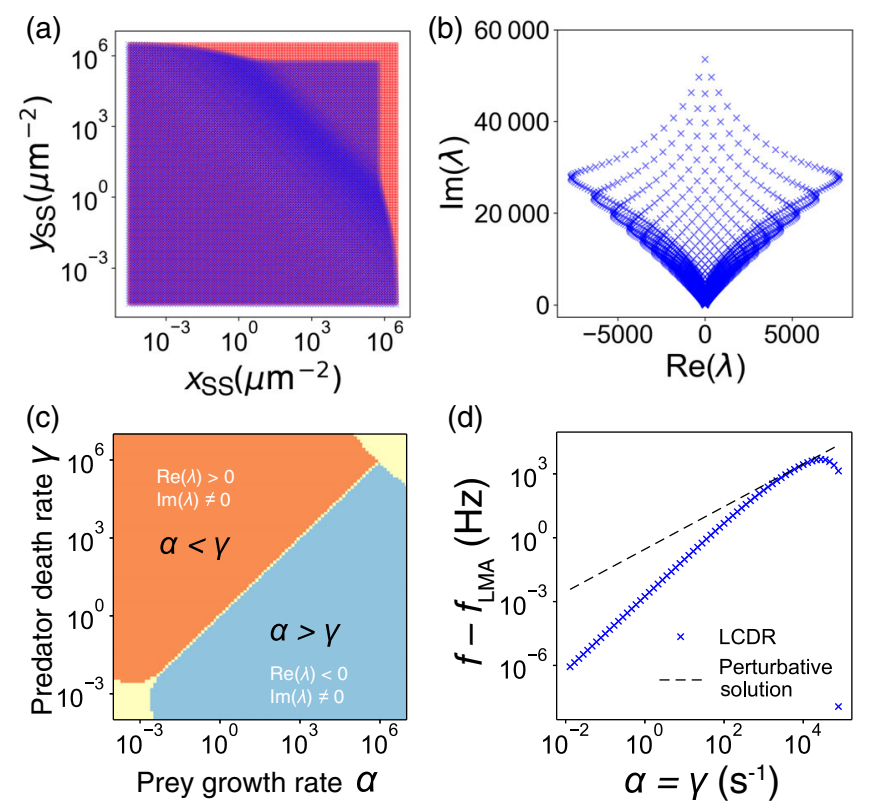

FIG. 6. Fine-tuning of oscillation: Solution of Lotka-Voterra predator-prey model using the LMA and LCDR leads to very different steady-state properties. (a) The steady-state prey $\left(x_{\mathrm{SS}}\right)$ vs predator $\left(y_{\mathrm{SS}}\right)$ concentration when the LMA is used (red dots) and when the LCDR is used (blue crosses). The compression of phase space shows that the LCDR constrains large variation in concentration, which is consistent with Fig. 5. (b) The real part of the eigenvalues of the Jacobian vs their imaginary parts shows that most steady-state solutions are nonoscillatory when the LCDR is used. (c) The phase diagram of the Lotka-Volterra (LV) predatorprey system. Under the LCDR, sustained oscillation of concentration (pale yellow) happens only when $\alpha=\gamma$ for a wide range of parameter values, which is a drastic reduction from the LMA, where oscillation occurs for all $\alpha$ and $\gamma$. (d) The deviation in the frequency of oscillation in the LCDR from that predicted by the LMA, $f-f_{\text {LMA }}$ vs $\alpha$ in the parameter range where oscillation happens only when $\alpha=\gamma$. Perturbation theory (see Appendix C) correctly captures the difference in a small range of $\alpha$ (dashed black line).

of $\alpha$ and $\gamma$. That is, the oscillation of the prey and the predator population is robust to the variation of the parameters. In the LCDR, we do see similar behavior when $\alpha, \gamma<10^{-2}$ or when $\alpha, \gamma>10^{5}$, where $\kappa(\Phi)$ is effectively $\Phi$ independent. However, outside these parameter ranges, the stability of the steady state depends on $\alpha$ and $\gamma$, as can be seen from the eigenvalues at different parameter values [Fig. 6(b)]. In particular, when $\alpha>\gamma$, the steady state is a stable fixed point. When $\alpha<\gamma$, the steady state is an unstable fixed point and admits exponential rise of the peak predator population and exponential fall of the prey population. Finally, only when $\alpha=\gamma$, the steady-state solution is periodic [Fig. 6(c)]. Therefore, in the LCDR, the parameters have to be fine-tuned to achieve sustained oscillation.

The frequency of the oscillating state is higher in the LCDR than in the LMA. A perturbative analysis of the LV 
equations supports this observation (Appendix C). In fact, its prediction agrees well for a range of values of $\alpha$ [Fig. 6(d)]. This analysis also predicts that the oscillation in the LV predator-prey model is extremely sensitive to the concentration dependence of rates: Even weak concentration dependence is sufficient to break the robustness of the oscillation. This result indicates that very special conditions are required for oscillatory reactions to remain stable, such as finite intrinsic reaction rate $\kappa_{I}$ or the presence of intrinsic or extrinsic noise.

\section{Stability of steady states in the presence of finite intrinsic reaction rates}

So far, we consider only the effect of the concentrationdependent reaction rate in the diffusion-limited regime. In this regime, the intrinsic reaction rate, $\kappa_{I} \rightarrow \infty$. However, in biological or physicochemical systems, we often encounter situations in which $\kappa_{I}$ is finite. Under such circumstances, the reaction kinetics may be entirely determined by the intrinsic reaction rates, which in turn, will restore the robust oscillatory behavior of the LV model. Therefore, we seek to understand how finite $\kappa_{I}$ modifies the phase diagram obtained in the diffusion-limited regime [Fig. 6(c)]. To do so, we define the effective predation rate $\beta_{\text {eff }}$ :

$$
\beta_{\mathrm{eff}}=\left(\frac{1}{\kappa_{I}}+\frac{1}{\beta(x, y)}\right)^{-1},
$$

where $\beta(x, y)$ is the concentration-dependent encounter rate. We use this effective predation rate to calculate the steady-state solutions of the LV equations and the stability of the solutions.

Following the classification described in Ref. [18], we investigate four different $\kappa_{I}$ values for which the transient kinetics are diffusion controlled $\left(100 \mu \mathrm{m}^{2} / \mathrm{s}\right)$, diffusion influenced $\left(10 \mu \mathrm{m}^{2} / \mathrm{s}\right)$, weakly diffusion influenced $\left(0.1 \mu \mathrm{m}^{2} / \mathrm{s}\right)$, and intrinsic reaction rate limited $\left(0.001 \mu \mathrm{m}^{2} / \mathrm{s}\right)$. We find that fine-tuned oscillation persists in all four cases, even when the transient kinetics is rate limited and diffusion plays a negligible role (Fig. 7). It happens because the stability of the steady-state behavior is not only determined by the effective predation rate but also its derivative at the steady state (see Appendix C). The derivative has non-negligible, albeit diminishing, influence even when $\kappa_{I} \ll D$, which leads to fine-tuned oscillation even when $\beta_{\text {eff }}$ is essentially concentration independent. Because of the diminishing influence of the derivative, the parameter space for fine-tuned oscillation shrinks with decreasing $\kappa_{I}$. This result suggests that the influence of the diffusive transport process can impact steady-state behavior even when it has negligible impact on the transient kinetics. In particular, in the context of our results, we find that fine-tuned oscillation is a robust behavior that persists even when the intrinsic reaction rates are several orders of magnitude smaller than the diffusion coefficient.
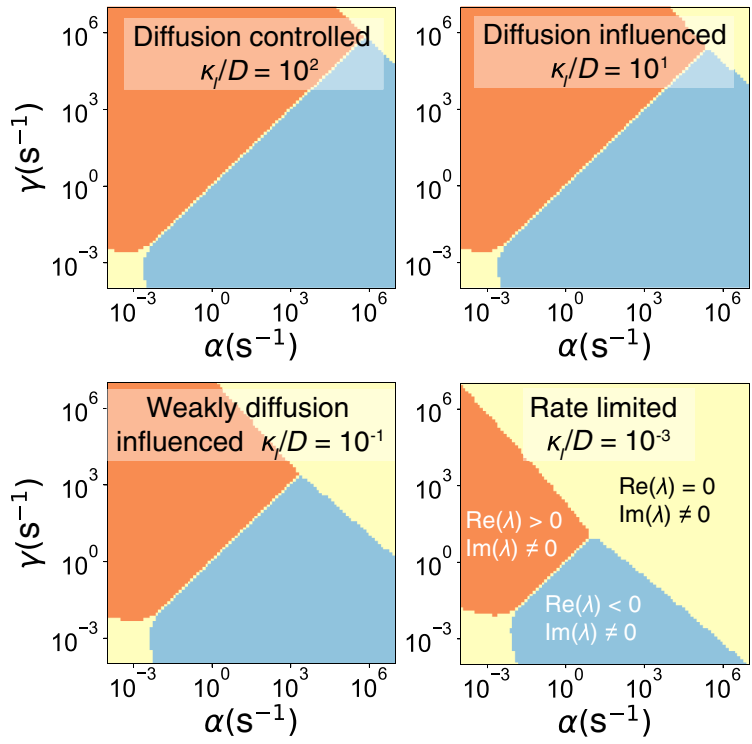

FIG. 7. Phase diagram of LV oscillators when the intrinsic reaction rate is finite for different intrinsic rates $\kappa_{I}$ at $D=1 \mu \mathrm{m}^{2} / \mathrm{s}$. We use the classification scheme provided in Ref. [18] to designate different regimes. We observe that finetuned oscillation persists even when the reactions are limited by the intrinsic reaction rates, although the corresponding parameter range narrows as $\kappa_{I}$ is decreased. These results suggest that the effect of diffusion may be felt even when the reaction rate is controlled almost entirely by the intrinsic rate $\kappa_{I}$.

\section{Intrinsic noise leads to the emergence of concentration- dependent rates}

The presence of intrinsic or extrinsic noise is an important determinant of the steady-state behavior, which we do not consider so far in this analysis. It is well known that noise can significantly change the behavior of chemical oscillators [44-48]. Its impact on the canonical LV systems, systems without concentration-dependent rates, have been widely studied. In particular, applying a chemical master equation for the birth-death processes to the LV reactions [Eq. (12)], it can be shown [49] that in the presence of intrinsic noise the mean concentrations of the predator and the prey follow modified deterministic equations:

$$
\begin{aligned}
& \frac{d x}{d t}=\alpha x-\beta_{0} x y-\beta_{0} G(x, y), \\
& \frac{d y}{d t}=\beta_{0} x y+\beta_{0} G(x, y)-\gamma y,
\end{aligned}
$$

where $G(x(t), y(t))=\operatorname{cov}[x(t), y(t)]$ is the (timedependent) covariance between the prey and the predator population. This equation has the same form as Eq. (13), with $\beta_{0} x y+\beta_{0} G(x, y)$ acting as the concentrationdependent predation propensity, $\beta(x, y) x y$. In particular, we find that for typical stochastic trajectories obtained from the canonical LV model [Fig. 8(a)], $G(x, y)$ has nonlinear dependence on $\Phi$. To see this dependence, we 

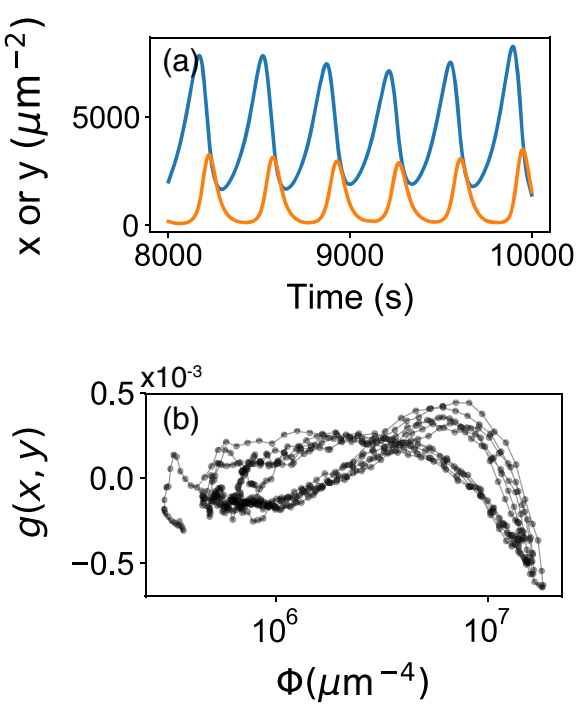

FIG. 8. Emergent concentration-dependent rates in the stochastic Lotka-Volterra equation: (a) A typical stochastic trajectory for $\alpha=0.01 \mathrm{~s}^{-1}, \quad \beta_{0}=0.00001 \mu \mathrm{m}^{2} \mathrm{~s}^{-1}$, and $\gamma=0.04 \mathrm{~s}^{-1}$, $x(t=0)=y(t=0)=1000 \mu \mathrm{m}^{-2}$. The blue line represents prey concentration $(x)$ and the orange line represents predator concentration (y). (b) For this trajectory, we measure the covariance per unit $\Phi, g(x, y)=G(x, y) / \Phi$ vs $\Phi . g(x, y)$ is the equivalent of $\kappa(\Phi)-\kappa_{0}$, and it has a nonlinear dependence on the mass action $\Phi$, which suggests that intrinsic noise can lead to concentrationdependent reaction rates.

define $g(x, y)=G(x, y) / \Phi$, which plays the same role in this context as $\kappa(\Phi)-\kappa_{0}$ does in the context of diffusioninduced concentration dependence. As Fig. 8(b) shows, $g(x, y)$ has a complex dependence on the mass action $\Phi$, which suggests that intrinsic noise may lead to the emergence of concentration-dependent reaction rates.

We must stress that the concentration dependence of $g(x, y)$ arises even when the predation rate $\beta_{0}$ is concentration independent. That is, the emergence of the concentration-dependent predation rate is not limited to dimensions less than three, and we can expect to observe its effect even in three dimension (3D). Moreover, even though the functional form of $g(x, y)$ is very different from $\beta(x, y)$, we expect the same drastic shrinkage of the parameter space available for stable oscillation of the concentrations, since the presence of any concentration dependence leads to fine-tuning of the oscillation (Appendix C). This observation may explain why it is difficult to obtain stable oscillation in the stochastic LV predator-prey model. However, more careful studies are needed to establish this claim.

Finally, here we show the effect of intrinsic noise only for cases when $\beta$ is not concentration dependent. It will be interesting to study the combined impact of the concentration-dependent predation rate $\beta(x, y)$ and the emergent rate $g(x, y)$ on the steady-state stability of the LV model and other dynamical systems. Moreover, it is likely that similar concentration-dependent rates will arise in biologically relevant situations also, which are noisy chemical systems. Therefore, a careful classification of the biological systems in terms of concentration dependence of the reaction rates is necessary. A classification scheme, in terms of accuracy of well-mixed reactions, has been suggested in Ref. [18]. However, as we show, we also need to consider the impact of diffusion and noise on such a classification.

\section{DISCUSSION}

\section{A. Concentration-dependent reaction rate}

In this paper, we use a hierarchical multiscale simulation framework to identify the origin of concentration dependence of the rate of association reactions and its consequences. We find that diffusion in 2D leads to the observed concentration dependence, and this dependence is universal across the different types of association reactions and the pair potentials considered here. In particular, the concentration-dependent rate $\kappa(\Phi)$ can be written in a simple empirical form, which leads to drastic changes in the steady-state properties of model chemical systems.

$\kappa(\Phi)$ is characterized by four parameters $\kappa_{0}, \kappa_{\max }, \epsilon$, and $\Phi_{0}$. When $\Phi \rightarrow 0, \kappa(\Phi) \rightarrow \kappa_{0}$, which is a concentrationindependent constant. $\kappa_{0}$ depends on the interaction potential, the reaction radius, and the diffusion coefficient. However, the diffusion coefficient merely rescales the value of $\kappa_{0}$ (see Fig. S3 in the Supplemental Material [30]), whereas the interaction potential and the reaction radius has more substantial impact. $\kappa_{0}$ depends on the interaction potential: $\kappa_{0}$ is higher for attractive LJ interaction than repulsive WCA interaction [Fig. 3(b)] for otherwise identical systems. Furthermore, we find that the lower the reaction radius, the lower the value of $\kappa_{0}$, which is what we expect [Fig. 3(b)]. In particular, when $r_{a}=0.89 r_{\min } \approx \sigma$, the value of $\kappa_{0}$ is comparable to its value computed using Smoluchowski's theory of diffusion-limited association reaction, which uses purely collision-based interaction between molecules [14,18] (Appendix D). For all analyses involving $\kappa(\Phi)$, we assume that $D=1 \mu \mathrm{m}^{2} / \mathrm{s}$ and $r_{a}=1.2 r_{\min }$, such that all $\kappa_{0}$ values are around $4 \mu \mathrm{m}^{2} / \mathrm{s}$. While the empirical laws fitted in Fig. 4(b) do depend on the value of $\kappa_{0}$, it does not make it less general. In fact, computation expense permitted, we can repeat the same set of analyses using $r_{a}=0.89 r_{\min }$, which has different $\kappa_{0}$ and $\Phi_{0}$ values but the same values of $\kappa_{\max }$ and $\epsilon$.

$\kappa_{\max }$ is the value of $\kappa(\Phi)$ in the other extreme, when $\Phi \rightarrow \infty$. As we discuss in Sec. II C, the value of $\kappa_{\max }$ can be measured by running simulations at higher packing fractions of the molecules, which we will do in a future paper. At higher packing fractions, the diffusive encounter rate is affected by the dynamic variation of the viscosity of the reactant solution. The variation of the viscosity depends on the interaction potential $[35,50]$. Therefore, we believe, the 
choice of attractive or repulsive interaction may lead to different $\kappa_{\max }$ values. However, in this paper, we ignore this difference. Instead, irrespective of the interaction potential, we use a mean-field formula derived using Smoluchowski's theory to estimate the value of $\kappa_{\max }$, which is around $20 \mu \mathrm{m}^{2} / \mathrm{s}$ (Appendix D).

Between $\kappa_{0}$ and $\kappa_{\max }, \kappa(\Phi)$ has a sigmoidal variation that is characterized by an exponent $\epsilon$ and a mass action scale $\Phi_{0}$. Through data analysis, we find that $\epsilon$ is equal to $3 / 8=0.375$. While we cannot provide a fundamental reason behind this exponent, the mean-field theory (Appendix D) predicts that for low $\Phi$ values, $\kappa(\Phi)-\kappa_{0}$ indeed scales as $\Phi^{0.4}$, which is remarkably close to what we find through our analysis. Therefore, it is likely that the $\Phi^{\epsilon}$ scaling emerges due to the properties of diffusion in $2 \mathrm{D}$.

The scale $\Phi_{0}$, in effect, measures the contribution of the concentration-dependent rates on the reaction kinetics. If $\Phi$ values are much lower than $\Phi_{0}$, the concentrationdependent rate is subdominant, and the behavior of the chemical system in this regime is well approximated by the LMA. However, for $\Phi$ values near or higher than $\Phi_{0}$, the reaction rates are strongly concentration dependent until they reach $\kappa_{\max }$, beyond which the rates become concentration independent again. Crucially, $\Phi_{0}$ is system dependent and higher values of $\Phi_{0}$ imply a broader range of validity of the LMA. For example, for the heterodimerization reaction, i.e., $A+B \rightarrow A B$, the $\Phi_{0}$ value is higher than that for the homodimerization reaction. Therefore, we expect the LMA to describe association reactions between unlike species much better than between like species.

We expect $\Phi_{0}$ to depend nontrivially on the form of the interaction potentials. In this paper, we consider only isotropic interactions of identical strength and measure their $\Phi_{0}$ values by fitting the functional form of $\kappa(\Phi)$ to the observed data. However, real systems often interact with each other through anisotropic interactions of varying strength. Understanding how $\kappa(\Phi)$ and $\Phi_{0}$ behave in these systems will help establish the range of validity of the LMA and render chemical kinetic models more accurate. In particular, it may be possible to estimate the values of $\kappa_{0}$ and $\Phi_{0}$ using self-consistent theories of association reaction in $2 \mathrm{D}[10,17,18,23,24]$.

\section{B. Dimensionality of concentration dependence}

In this paper, the concentration dependence of the reaction rates stems from the peculiarities of diffusion in 2D. The reentrant nature of diffusion in dimensions lower than three leads to the observed concentration dependence, which, as we expose, leads to drastic changes in the steadystate properties of dynamical systems. However, concentration dependence can originate from other factors as well, which we do not consider here. For example, viscosity can change dynamically at molecular packing fractions that are quintessentially found inside a cell. Such dynamic changes in viscosity can dramatically reduce both translational and rotational diffusion coefficients [35], which can give rise to another form of concentration dependence of the diffusionlimited reaction rates. Such crowding-induced concentration dependence is not limited by the dimension of the problem and can be observed even in 3D [20]. Furthermore, as we show, albeit nonrigorously, intrinsic noise can also lead to emergent concentration dependence of the association reaction rates, even when the original reaction rates are concentration-independent constants. It is well known that diffusion-limited reaction rates are concentrationindependent constants only for dimensions greater than or equal to three. Therefore, intrinsic noise offers another mechanism to obtain concentration-dependent reaction rates in 3D. We expect that these three different flavors of concentration dependence will have different impacts on the transient kinetics and may even have different steadystate behaviors. However, all of these claims warrant rigorous examination, which we hope will inspire many future investigations of the origin and the consequences of the concentration-dependent rates.

\section{Impact on biomolecular systems}

Concentration dependence of the reaction rates leads to drastic changes in the behavior of simple chemical systems, which may have serious repercussions on the behavior of more complex chemical systems, such as the cell-signaling or metabolic reactions. We find that concentrationdependent reaction rates lead to a reduction in intrinsic noise in dimerization reaction. Crucially, they destabilize robust oscillation in the LV predator-prey system and render it fine-tuned to the parameter values. Although the LV model is less relevant for biomolecular systems, the results derived from this model system can be directly applied to study feedback-driven oscillatory systems on cell membranes or other 2D surfaces inside the cell. For example, signaling circuits in cell growth and development do show oscillatory behavior $[8,9,29,51,52]$. These circuits are usually membrane bound. Therefore, we expect our results to be directly applicable to such systems.

The concentration-dependent rates have another important implication in the context of modeling of biomolecular systems. The systems biology models used to study complex biological functions, such as cell signaling or metabolism, often use reaction rates measured from experiments. More often than not, the measured reaction rates vary wildly over several orders of magnitude [53]. Although such variations are often attributed to experimental imprecision, our results offer a plausible alternate hypothesis. As we see, the concentration-dependent rates can vary across several orders of magnitude. Also, it is well known that cells modulate the concentration of the biomolecules to achieve different tasks. It is possible that the rates measured by different experiments have different concentration of the reactant molecules, which leads to the broad variation of the measured rates. This hypothesis 
has two implications. First, it implies that the larger the range of reaction rates, the larger is the concentration fluctuation of the reacting molecules. Second, it will be possible to fit a sigmoidal $\kappa(\Phi)$ using the measured rates and use that concentration dependence to construct a class of systems biology models. Given that the concentrationdependent reaction rates have such drastic impact on the simple systems, it will lead to very interesting and potentially undiscovered phenomena in complex chemical systems.

\section{ACKNOWLEDGMENTS}

This work is funded by LDRD Grant No. XX0100 from LANL and this document has the following LA-UR number: LA-UR-20-24037. Computations use resources provided by the LANL Institutional Computing Program, which is supported by the U.S. DOE National Nuclear Security Administration under Contract No. DE-AC5206NA25396. The author thanks Professor Angel E. Garcia, Dr. Van A. Ngo, Professor Yair Shokef, and Dr. Sumit Majumder for useful discussion and critical reading of the manuscript. The author also thanks two anonymous referees whose input has significantly improved the quality of this paper.

\section{APPENDIX A: METHODS}

\section{Molecular simulation}

We use a recently developed molecular simulation technique called GFRD [13,25-28] to perform molecular simulation. The basic tenet of the GFRD is that tagged particles, such as proteins, in biomolecular systems can be partitioned into two groups: (a) isolated particles, which freely diffuse and (b) interacting particles, which interact with other particles. In a special form of the GFRD, the isolated particles are propagated in an event-driven manner using the Green's function of diffusion until the particle encounters another particle and it can no longer be treated as an isolated particle, whereas the interacting particles are propagated using a molecular mechanics algorithm, such as molecular dynamics, dissipative particle dynamics, or Markov state models [25]. In the present work, we use overdamped $\mathrm{BD}$ as our molecular mechanics algorithm. This updated form is called BD-GFRD $[25,26]$.

We assume that the particles of type $A$ are spheres of radius $2 \mathrm{~nm}$ and particles of type $B$ are of radius $2.9 \mathrm{~nm}$ [Fig. 1(b)], and they interact with each other through shortranged isotropic interactions, such as the 6-12 LennardJones or WCA interaction [Fig. 1(c)] of strength $6 k_{B} T$, where $k_{B}$ is Boltzmann's constant and $T=310 \mathrm{~K}$ is the temperature of the heat bath. In addition, the diffusion constants of particles of type $A$ and type $B$ are $1 \mu \mathrm{m}^{2} / \mathrm{s}$ and $0.69 \mu \mathrm{m}^{2} / \mathrm{s}$, respectively. Because we assume overdamped dynamics, their mass is irrelevant for the simulation. These parameter values correspond to typical protein-protein interaction parameters, e.g., Ras-Raf interaction [33]. Furthermore, we assume that the reactions happen on a 2D surface, such as the plasma membrane of a cell. Hence, all of our BD-GFRD simulations are done on a $1 \times 1 \mu \mathrm{m}^{2}$ plane with periodic boundary conditions. Using this setup, we run the simulations for $96 \mathrm{CPU}$ hours or $10^{4}$ dimerization events, whichever occurs first. We reach a timescale of about $100 \mathrm{~ms}$ for $300 / \mu \mathrm{m}^{2}$, the largest concentration we consider here.

While using spherical particles is necessary to use the current BD-GFRD framework, the interaction potential need not be isotropic. However, for the ease of exposition, we use isotropic potential in this paper. We study reaction kinetics under both attractive and repulsive potential. We model the former potential using a $\mathrm{LJ}$ potential with cutoff at $2.5 \sigma$ and the latter potential with a WCA interaction [54] [Fig. 1(c)]. In this paper, we report the results for cases when the interaction strength for both potentials is $6 k_{B} T$. However, we check our results for various interaction strengths up to $10 k_{B} T$ and find that the variation of interaction energies within this range does not affect our results. The length scale of the interaction potentials $\sigma$ is chosen to be equal to the sum of the radius of the two reactants.

We consider two different types of association reactions: homodimerization and heterodimerization. For the former reaction, we consider reactions of type $A+A \rightarrow A_{2}$, while for the latter reaction, we consider reactions of the form $A+B \rightarrow A B$. To simulate association reaction, we use a special case of Doi's volume reaction model $[31,32]$. We assume that the particles react as soon as their distance becomes less than or equal to the reaction distance $r_{a}$ [Fig. 1 (d)]. The reaction radius can be larger or smaller than $r_{\min }$, the location of the minimum of the interaction potential. Based on our previous work, unless otherwise stated, we assume that $r_{a}=1.2 r_{\min }$. We find that the concentration dependence does not depend qualitatively on $r_{a}$, but it has quantitative impact, which we discuss in this paper.

\section{Measurement of $\boldsymbol{\kappa}(\boldsymbol{\Phi})$}

We measure the reaction rates in an ensemble, in which the total number of particles of all species remains constant. We start with all monomers and no homo- or heterodimers. Hence, we remove a product molecule from the simulation as soon as it forms and replace it with the reactant molecules that it is formed from. We place the reactant molecules randomly on the simulation box to avoid introduction of unwanted correlation. Because we consider only dilute systems, such random replacements very rarely encounter another molecule after the replacement and, hence, does not break detailed balance. Furthermore, if there is such an encounter, we reject the random replacement and repeat the random placement until there is no encounter. In fact, this process is equivalent to starting with a new initial condition after each dimerization event. For denser systems, which we do not consider in this paper, we 
have to use sampling techniques, such as the metropolis algorithm, that preserve detailed balance.

To measure the reaction rates, we compute the probability distribution function (PDF) of the time interval between two product formation reactions. The reaction propensities $r$ are then calculated by fitting the exponential tail of the PDFs. The exponential tail results from the reactions, whose rates are lower than the diffusive encounter rate. Therefore, we can use a well-mixed approximation, i.e., Eq. (7), to calculate $\kappa(\Phi)$ :

$$
r=\kappa(\Phi) \Phi .
$$

\section{Chemical kinetic model}

We use $\kappa(\Phi)$ to construct chemical kinetic models that are solved using the Gillespie algorithm or analytical calculation. For the dimerization reactions, each stochastic simulation is run until the simulation time reaches $t_{\max }=$ $100 \mathrm{~s}$ or $100 / k_{\text {off }}$, whichever is shorter. The steady-state values are measured by averaging the observables between $t_{\max } / 2$ and $t_{\max }$ over 100 different replicates. The system reaches a steady state within $t_{\max } / 10$ for all parameter values explored. The chemical kinetic equation for the product concentration $P$ is given by

$$
\begin{aligned}
\frac{d P}{d t} & =k_{\mathrm{on}}-k_{\mathrm{off}}[P] \\
& =\kappa(\Phi) \Phi-\kappa(\Phi) k_{D}[P] .
\end{aligned}
$$

In this equation, $k_{\mathrm{on}}=r(\Phi)=\kappa(\Phi) \Phi$ is the propensity of the concentration-dependent diffusion-influenced reaction, and $\Phi$ is the mass action of the reaction. For the LMA, $k_{\text {on }}$ is a $\Phi$-independent constant, whereas for the LCDR, $k_{\text {on }}$ depends on $\Phi . k_{\text {off }}$ is the dissociation rate. For reversible reactions approaching chemical equilibrium, $k_{\text {off }}$ is given by the product of the association rate $k_{\text {on }}$ and the equilibrium constant $k_{D}$, which ensures detailed balance. For nonequilibrium steady states, $k_{\text {off }}$ are varied independently from $k_{\mathrm{on}}$.

\section{APPENDIX B: STEADY-STATE CONCENTRATION OF MOLECULES IN DIMERIZATION REACTION}

\section{Equation for steady-state concentration}

Let us consider the following dimerization reaction:

$$
A+A \underset{k_{\text {off }}}{\stackrel{k_{\text {on }}}{\rightleftharpoons}} A_{2} .
$$

The concentrations of the molecules obey the following set of kinetic equations:

$$
\begin{gathered}
\frac{d[A]}{d t}=-2 k_{\mathrm{on}} \Phi+2 k_{\mathrm{off}}\left[A_{2}\right], \\
\frac{d\left[A_{2}\right]}{d t}=k_{\mathrm{on}} \Phi-k_{\mathrm{off}}\left[A_{2}\right] . \\
\Phi=\frac{[A]([A]-1)}{2}, \\
k_{\mathrm{on}}= \begin{cases}k_{0} & \text { for LMA, } \\
\kappa(\Phi) & \text { for LCDR, }\end{cases} \\
\kappa(\Phi)=\frac{\kappa_{\max } \kappa_{0}}{\kappa_{0}+\left(\kappa_{\max }-\kappa_{0}\right) \exp \left[-\left(\frac{\Phi}{\Phi_{0}}\right)^{\epsilon}\right]} .
\end{gathered}
$$

In steady state, the following relation holds:

$$
k_{\text {on }} \Phi=k_{\text {off }}\left[A_{2}\right] .
$$

In addition, we assume that the total number of monomers $N$ is fixed, such that

$$
[A]+2\left[A_{2}\right]=N .
$$

Combining Eqs. (B7) and (B8), we get the following equation, whose roots are the steady-state concentrations of the monomers,

$$
k_{\text {on }} \times x(x-1)-k_{\text {off }}(N-x)=0,
$$

where $x=[A]$ is the steady-state concentration of the monomer and $\Phi=x(x-1) / 2$ is the mass action.

\section{Solution}

The solution of Eq. (B9) depends on the nature of the steady state. For example, if the steady state is at chemical equilibrium, then $k_{\text {off }}$ has to be equal to $k_{\text {on }} k_{D}$ at all times, where $k_{D}$ is the equilibrium constant. As a result, for both the LMA and LCDR, Eq. (B9) simplifies to

$$
x(x-1)-k_{D}(N-x)=0,
$$

which has a simple solution:

$$
x_{\mathrm{Eq}}=\frac{\sqrt{\left(k_{D}-1\right)^{2}+4 k_{D} N}-\left(k_{D}-1\right)}{2} .
$$

However, when the steady state is a nonequilibrium steady state, $k_{\text {off }}$ does not have to be exactly equal to $k_{\text {on }} k_{D}$; we can control it independently from $k_{\text {on }}$. For the sake of simplicity, let us assume that $k_{\text {off }}=k_{D}$. Furthermore, we assume that under the LMA $k_{\text {on }}=k_{0}$, where $k_{0}$ is a constant, from which we find that the solution at the nonequilibrium steady state is 
(a)

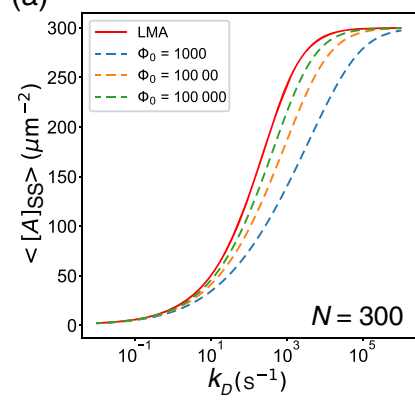

FIG. 9. Variation of steady-state monomer concentration with $k_{D}$ for (a) $N=300$ and (b) $N=1000 . \kappa_{0}=1$, and $\kappa_{\max }=1000$ were used for all plots. $\Phi_{0}$ values for the LCDR cases are shown in the legend. $k_{0}=1$ is used for the LMA solution.

$$
\begin{aligned}
x_{\mathrm{MA}} & =\frac{\sqrt{\left(k_{D}-k_{0}\right)^{2}+4 k_{D} k_{0} N}-\left(k_{D}-k_{0}\right)}{2 k_{0}}, \\
& =\frac{\sqrt{\left(k_{D}^{\mathrm{SC}}-1\right)^{2}+4 k_{D}^{\mathrm{SC}} N}-\left(k_{D}^{\mathrm{SC}}-1\right)}{2},
\end{aligned}
$$

where $k_{D}^{\mathrm{SC}}$ is equal to $k_{D} / k_{0}$. It is evident from this solution that, under the LMA, it is not possible to attain a nonequilibrium steady state by manipulating $k_{\text {off }}$. Doing so merely scales $k_{D}$ to a different value, and the steady-state fluctuations are equilibrium fluctuations that obey detailed balance.

In contrast, when the LCDR is used it is possible to break detailed balance by manipulating $k_{\text {off }}$. To show broken detailed balance, we compute the steady-state solution using $k_{\mathrm{on}}=\kappa(\Phi)$ and $k_{\mathrm{off}}=k_{D}$. Unfortunately, the closed-form solution is not easy to obtain, so we use the Newton-Raphson root-finding algorithm to find the solutions of Eq. (B9). Steady-state solutions for a few $\Phi_{0}$ and $N$ are shown in Figs. 9(a) and 9(b). Clearly, the nonequilibrium steady-state solutions are different for hte LCDR than the equilibrium solution that we obtain for both the LMA and LCDR. In general, the higher the $\Phi_{0}$, the more similar the LCDR and LMA solutions are. Physically, it implies that the validity of the LMA increases as the difficulty for an association reaction increases. If the association reaction is a rare event, then the reaction events will follow Poisson distribution. Hence, the reaction rate will be a concentration-independent constant.

\section{APPENDIX C: STEADY-STATE BEHAVIOR OF THE LOTKA-VOLTERRA MODEL}

We study the LV predator-prey equations [16], which describe the dynamics of the following set of reactions:

$$
\begin{gathered}
X \rightarrow 2 X, \\
X+Y \rightarrow 2 Y,
\end{gathered}
$$

$$
Y \rightarrow \phi
$$

where $X$ is the prey, $Y$ is the predator, and $\phi$ represents death. The most general LV equation can be written in the following way:

$$
\begin{aligned}
& \frac{d x}{d t}=\alpha x-\beta(x, y) x y, \\
& \frac{d y}{d t}=\beta(x, y) x y-\gamma y,
\end{aligned}
$$

where $\alpha$ is the birth rate of the prey, $\beta(x, y)$ is a concentration-dependent predation rate, and $\gamma$ is the death rate of the predator. The equation for the steady-state concentrations is defined by the following two equations [55]:

$$
\begin{aligned}
& y=\alpha / \beta(x, y), \\
& x=\gamma / \beta(x, y) .
\end{aligned}
$$

The Jacobian of Eq. (C4) is

$$
J(x, y)=\left(\begin{array}{cc}
\alpha-\frac{\partial \beta}{\partial x} x y-\beta y & -\frac{\partial \beta}{\partial y} x y-\beta x \\
\frac{\partial \beta}{\partial x} x y+\beta y & \frac{\partial \beta}{\partial y} x y+\beta x-\gamma
\end{array}\right) .
$$

\section{Steady-state behavior under the LMA}

When the LMA is used, $\beta(x, y)=\beta_{0}$. Therefore, the steady-state solutions are

$$
\begin{aligned}
& y=\alpha / \beta_{0}, \\
& x=\gamma / \beta_{0},
\end{aligned}
$$

and the Jacobian at this steady-state value is

$$
J(x, y)=\left(\begin{array}{cc}
0 & -\gamma \\
\alpha & 0 .
\end{array}\right)
$$

Therefore, for all $\alpha>0$ and $\gamma>0$, the eigenvalues are $\pm l \sqrt{\alpha \gamma}$; i.e., the steady-state solution is oscillating, and it is robust to perturbation in $\alpha$ and $\gamma$.

\section{Steady-state behavior under the LCDR}

a. General steady-state solution

When the LCDR is used, $\beta(x, y)=\kappa(\Phi)$ for $\Phi=x y$. $\kappa(\Phi)$ is defined in Eq. (B6). Equations (C6) and (C7) can be used to write the following equation for $\Phi$ :

$$
\Phi \beta^{2}(\Phi)=\alpha \gamma
$$

By dividing both sides by $\Phi_{0}$ and defining $z=\Phi / \Phi_{0}$, we write the reduced equation for the steady-state solution, 


$$
z \beta^{2}(z)-\frac{\alpha \gamma}{\Phi_{0}}=0
$$

If $z_{\mathrm{SS}}$ is the solution of this equation, then the steady-state concentrations of the prey and predator are

$$
\begin{aligned}
& x_{\mathrm{SS}}=\gamma / \beta\left(z_{\mathrm{SS}}\right), \\
& y_{\mathrm{SS}}=\alpha / \beta\left(z_{\mathrm{SS}}\right) .
\end{aligned}
$$

The Jacobian can also be easily constructed by noting that $(\partial \beta / \partial x$ or $y)=\left(x\right.$ or $\left.y / \Phi_{0}\right)(\partial \beta / \partial z)$.

\section{b. Perturbation theory}

We can gain some insights into the stability of the steadystate solution by solving Eqs. (C6) and (C7) using perturbation theory. To do so, we note that near $z=0$, $\beta(z) \approx \kappa_{0}+\kappa_{1} z^{\epsilon} \approx \beta_{1} \Phi^{\delta}=\beta_{1}(x y)^{\delta}$. In general, $\delta$ is a function of $\Phi$, and $\beta_{1}$ is a constant. In particular, $\delta \rightarrow 0$ as $\Phi \rightarrow 0$, and $\delta \rightarrow \epsilon$ for $\Phi \gg \Phi_{0}$. Under this approximation, the steady-state solutions are

$$
\begin{gathered}
\Phi_{\mathrm{SS}}=\left(\frac{\alpha \gamma}{\beta_{1}^{2}}\right)^{\frac{1}{2 \delta+1}}, \\
\beta\left(x_{\mathrm{SS}}, y_{\mathrm{SS}}\right)=\beta_{1}^{1 / 1+2 \delta} \times(\alpha \gamma)^{\delta / 1+2 \delta}, \\
x_{\mathrm{SS}}=\frac{\gamma}{\beta\left(x_{\mathrm{SS}}, y_{\mathrm{SS}}\right)}, \\
y_{\mathrm{SS}}=\frac{\alpha}{\beta\left(x_{\mathrm{SS}}, y_{\mathrm{SS}}\right)} .
\end{gathered}
$$

In particular, when $\alpha=\gamma$, their values are

$$
x_{\mathrm{SS}}=y_{\mathrm{SS}}=\left(\frac{\gamma}{\beta_{1}}\right)^{1 /(1+2 \delta)},
$$

which scale as $\gamma$ when $\Phi \rightarrow 0$, such that $\delta \rightarrow 0$, and scale as $\gamma^{1 /(1+2 \epsilon)}$ when $\Phi \sim \Phi_{0}$ or higher.

The Jacobian is

$$
J(x, y)=\left(\begin{array}{cc}
\alpha-y \beta(x, y)(1+\delta) & -x \beta(x, y)(1+\delta) \\
y \beta(x, y)(1+\delta) & x \beta(x, y)(1+\delta)-\gamma
\end{array}\right),
$$

which in the steady state has the simple form

$$
J\left(x_{\mathrm{SS}}, y_{\mathrm{SS}}\right)=\left(\begin{array}{cc}
-\alpha \delta & -\gamma(1+\delta) \\
\alpha(1+\delta) & \gamma \delta
\end{array}\right) .
$$

The eigenvalues of this Jacobian satisfy the following characteristic equation:

$$
(\lambda-\delta \gamma)(\lambda+\delta \alpha)+(1+\delta)^{2} \alpha \gamma=0,
$$

$$
\Rightarrow \lambda^{2}+\delta(\alpha-\gamma) \lambda+(1+2 \delta) \alpha \lambda=0 .
$$

Therefore, the eigenvalues are

$\lambda_{ \pm}=-\frac{(\alpha-\gamma) \delta}{2} \pm \frac{\sqrt{\delta^{2}(\alpha-\gamma)^{2}-4(1+2 \delta) \alpha \gamma}}{2}$.

When $\delta=0$, we recover the robust oscillatory behavior, as expected from the LMA. However, as soon as $\delta>0$, oscillation happens only when $\alpha=\gamma$. It is a drastic shrinkage of the parameter space compared to the LMA. In fact, in the LCDR, to get sustained oscillation, the parameters have to be fine-tuned such that $\alpha=\gamma$. Moreover, when oscillation happens, it happens with a frequency of $\sqrt{(1+2 \delta) \alpha \gamma}$, which is $\sqrt{1+2 \delta}$ times faster than the frequency of oscillation in the LMA.

When $\alpha>\gamma$, the real part of the eigenvalues are negative, and the steady state is a stable fixed point. On the other hand, when $\gamma>\alpha$, the real part of the eigenvalues is greater than zero, the steady state is unstable to perturbations, the predator population grows exponentially, and the prey populations shrink exponentially, eventually resulting in the extinction of the both prey and predator population. However, one should note that true collapse of the population is not possible in an ordinary-differentialequation-based model because $x=0, y=0$ is a saddle point, which means that the population can recover as long as it is greater than 0 .

When $\alpha \neq \gamma$, the population may reach its steady state through oscillation or without oscillation. The crossover between these two regions happens when the imaginary part of the eigenvalues becomes 0 . That is when

$$
\begin{aligned}
\delta^{2}(\alpha-\gamma)^{2} & =4(1+2 \delta) \alpha \gamma, \\
& \Rightarrow \gamma=\frac{\eta+1}{\eta-1} \alpha, \quad \text { when } \alpha<\gamma, \\
& =\frac{\eta-1}{\eta+1} \alpha \quad \text { when } \alpha>\gamma, \\
\eta & =\sqrt{\frac{(1+\delta)^{2}}{1+2 \delta}} .
\end{aligned}
$$

The crossover region is defined by a line with slope $m=(\eta \pm 1 / \eta \mp 1)$. However, we note that $\delta$ increases with $\Phi$. Hence, $m$ changes with $\Phi$. At large enough $\Phi$, $m \approx 1$, whereas when $\Phi$, hence $\delta$, is small, the slope $m \rightarrow$ $\infty$ or $\rightarrow 0$, depending on whether $\alpha<\gamma$ or $\alpha>\gamma$, respectively. We can combine the preceding analysis to find the steady-state solution of the oscillatory state (Fig. 10) and construct a phase diagram, which is shown in Fig. 11. 


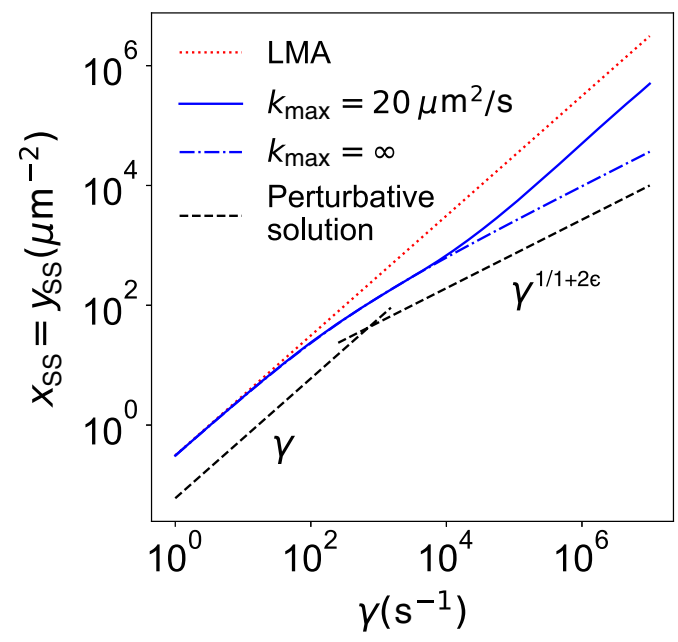

FIG. 10. The steady-state concentration of the oscillatory state is given by Eq. (C18) when $\gamma=\alpha$. The LMA steady state increases linearly with $\gamma$ (red dotted line), but the LCDR steady state increases sublinearly except for $\gamma \approx 0$ (blue solid line) for both $k_{\max }=20 \mu \mathrm{m}^{2} / \mathrm{s}$ and $k_{\max }=\infty$. For $k_{\max }=\infty$, such that $\kappa(\Phi)$ is always $\kappa_{0}+\kappa_{1} \Phi^{\epsilon}$, the LCDR steady-state solution is correctly given by the perturbation theory (black dashed line), which predicts that $x_{\mathrm{SS}}$ and $y_{\mathrm{SS}}$ scale as $\gamma$, when $\Phi$; i.e., $x_{\mathrm{SS}}$ is small and they scale as $\gamma^{1 / 1+2 \epsilon}$ when $\Phi>\Phi_{0}=1 \times 10^{5}$.

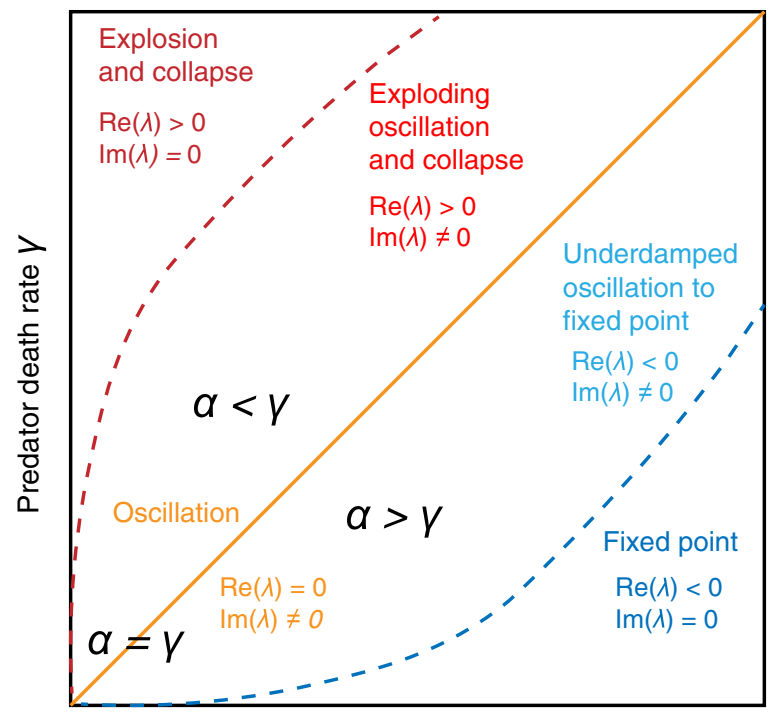

Prey growth rate $\alpha$

FIG. 11. Phase diagram obtained using the perturbation theory. Perturbation theory correctly predicts the phase boundary between stable fixed points, unstable fixed points, and sustained oscillation ( $\alpha=\gamma$, yellow line). However, it incorrectly predicts the phase boundaries that distinguish the oscillating from nonoscillating approach to the fixed points (broken blue and crimson lines) at small $\alpha$ and $\gamma$. A key drawback of the perturbation theory is that it does not account for the sigmoidal nature of $\kappa(\Phi)$ and its saturation at $\kappa_{\max }$, which removes the nonoscillating regions (explosion and collapse and fixed point) from the phase diagram.

\section{APPENDIX D: COMPUTATION OF $\kappa_{\max }$ USING SMOLUCHOWSKI THEORY}

The concentration-dependent reaction rate in the presence of collisional interaction is given by

$$
\kappa=8 \pi D\left(\frac{4 \log \frac{b}{\sigma}}{\left(1-\sigma^{2} / b^{2}\right)^{2}}-\frac{2}{\left(1-\sigma^{2} / b^{2}\right)}-1\right)^{-1},
$$

where $D$ is the diffusion coefficient, $\sigma$ is the sum of the radius of two reacting molecules, and $b$ is the average radius of a circular region where only one reaction is possible [18]. Clearly, $b$ is a concentration-dependent quantity, and the higher the concentration of the reactants, the lower the value of $b$. In fact, we can estimate $b$ as a function of the concentration using a mean-field approximation. If the mass action is $\Phi$ and the area of the simulation box is $A$, then on average, the area per reaction is $A / \Phi$, and $b$ is given by [18]

$$
b(\Phi)=2 \sqrt{\frac{A}{\pi \Phi}-r_{1}^{2}-r_{2}^{2}},
$$

where $r_{1}$ and $r_{2}$ are the radii of the two interacting molecules. Plugging this expression in Eq. (D1) for $A_{2}$, we get Fig. 12, which shows that $\kappa_{\max } \approx 20$ [Fig. 12(a)] and $\epsilon$ is approximately 0.4 for small $\Phi$ values [Fig. 12(b)]. Furthermore, $\kappa_{0}$ can be estimated by extrapolating Eq. (D1) to the $\Phi \rightarrow 0$ limit. Practically, it is estimated by finding the value of $\kappa / D$ at $\Phi=1$, which is approximately 1.2 [Fig. 12(a)].

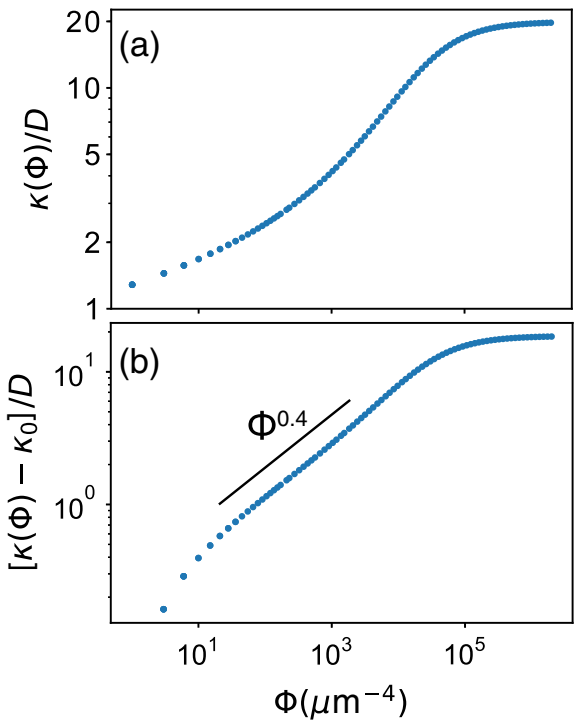

FIG. 12. $\quad \kappa(\Phi)$ obtained using Smoluchowski theory with meanfield approximation. (a) $\kappa(\Phi)$ vs $\Phi$ shows that $\kappa(\Phi) / D$ saturates at around 20, which we take to be the value of $\kappa_{\max }$. The value of $\kappa_{0} / D$ is between 1 and 2. (b) $\kappa(\Phi)-\kappa_{0}$ scales as $\Phi^{0.4}$ for small $\Phi$ values. 
The value of the exponent derived from the mean-field theory is remarkably close to the values found through the analysis of the simulation data, but it is not exactly equal to what we find. This difference stems from the fact that $A / \Phi$ underestimates the area available for each reaction. In reality, the area available varies as $A / \Phi^{\alpha}$, where $\alpha<1$. We find that as $\alpha$ is lowered from $1, \epsilon$ computed using Eq. (D1) also decreases. Therefore, for a suitable $\alpha$, the value of $\epsilon$ will be 0.375 . However, we need careful investigation of the diffusion-limited reactions to identify such an $\alpha$.

[1] P. Atkins, J. de Paula, and J. Keeler, Atkins' Physical Chemistry 11e, 11th ed. (Oxford University Press, New York, 2018).

[2] A. M. Turing, The Chemical Basis of Morphogenesis, Phil. Trans. R. Soc. B 237, 37 (1952).

[3] M. C. Cross and P. C. Hohenberg, Pattern Formation outside of Equilibrium, Rev. Mod. Phys. 65, 851 (1993).

[4] A.-L. Barabási and H. E. Stanley, Fractal Concepts in Surface Growth (Cambridge University Press, Cambridge, England, 1995).

[5] L. A. Chylek, D. A. Holowka, B. A. Baird, W. S. Hlavacek, D. A. Holowka, B. A. Baird, and W. S. Hlavacek, Quantitative Modeling of Mast Cell Signaling (Taylor \& Francis, London, 2018).

[6] B. Harmon, L. A. Chylek, Y. Liu, E. D. Mitra, A. Mahajan, E. A. Saada, B. R. Schudel, D. A. Holowka, B. A. Baird, B. S. Wilson, W. S. Hlavacek, and A. K. Singh, Timescale Separation of Positive and Negative Signaling Creates History-Dependent Responses to IgE Receptor Stimulation, Sci. Rep. 7, 1 (2017).

[7] L. A. Chylek, V. Akimov, J. Dengjel, K. T. G. Rigbolt, B. $\mathrm{Hu}, \mathrm{W} . \mathrm{S}$. Hlavacek, and B. Blagoev, Phosphorylation Site Dynamics of Early T-Cell Receptor Signaling, PLoS One $\mathbf{9}$, e104240 (2014).

[8] U. Alon, An Introduction to Systems Biology: Design Principles of Biological Circuits (CRC Press, Boca Raton, FL, 2019).

[9] J. R. Karr, J. C. Sanghvi, D. N. Macklin, M. V. Gutschow, J. M. Jacobs, B. Bolival, N. Assad-Garcia, J. I. Glass, and M. W. Covert, A Whole-Cell Computational Model Predicts Phenotype from Genotype, Cell 150, 389 (2012).

[10] A. Szabo, K. Schulten, and Z. Schulten, First Pssage Time Approach to Diffusion Controlled Reactions, J. Chem. Phys. 72, 4350 (1980).

[11] N. Agmon and A. Szabo, Theory of Reversible DiffusionInfluenced Reactions, J. Chem. Phys. 92, 5270 (1990).

[12] R. W. Pastor, R. Zwanzig, and A. Szabo, Diffusion Limited First Contact of the Ends of a Polymer: Comparison of Theory with Simulation, J. Chem. Phys. 105, 3878 (1996).

[13] K. Takahashi, S. Tanase-Nicola, and P. R. ten Wolde, Spatio-Temporal Correlations Can Drastically Change the Response of a MAPK Pathway, Proc. Natl. Acad. Sci. U.S.A. 107, 2473 (2010).
[14] M. Dibak, C. Fröhner, F. Noé, and F. Höfling, DiffusionInfluenced Reaction Rates in the Presence of Pair Interactions, J. Chem. Phys. 151, 164105 (2019).

[15] T. G. Kurtz, The Relationship between Stochastic and Deterministic Models for Chemical Reactions, J. Chem. Phys. 57, 2976 (1972).

[16] J. D. Murray, Mathematical Biology: I. An Introduction (Springer Science \& Business Media, New York, 2007), Vol. 17.

[17] D. C. Torney and H. M. McConnell, Diffusion-Limited Reaction Rate Theory for Two-Dimensional Systems, Proc. R. Soc. A 387, 147 (1983).

[18] O. N. Yogurtcu and M. E. Johnson, Theory of Bi-Molecular Association Dynamics in 2D for Accurate Model and Experimental Parameterization of Binding Rates, J. Chem. Phys. 143, 084117 (2015).

[19] R. Grima and S. Schnell, How Reaction Kinetics with TimeDependent Rate Coefficients Differs from Generalized Mass Action, Chem. Phys. Chem. 7, 1422 (2006).

[20] R. Grima and S. Schnell, A Systematic Investigation of the Rate Laws Valid in Intracellular Environments, Biophys. Chem. 124, 1 (2006).

[21] M. O. Vlad, A. D. Corlan, F. Morán, R. Spang, P. Oefner, and J. Ross, Kinetic Laws, Phase-Phase Expansions, Renormalization Group, and INR Calibration, Proc. Natl. Acad. Sci. U.S.A. 106, 6465 (2009).

[22] B. Boros, J. Hofbauer, S. Müller, and G. Regensburger, The Center Problem for the Lotka Reactions with Generalized Mass-Action Kinetics, Qual. Theory Dyn. Syst. 17, 403 (2018).

[23] A. Szabo, Theory of Diffusion-Influenced Fluorescence Quenching, J. Phys. Chem. 93, 6929 (1989).

[24] I. V. Gopich, Multisite Reversible Association in Membranes and Solutions: From Non-Markovian to Markovian Kinetics, J. Chem. Phys. 152, 104101 (2020).

[25] A. Vijaykumar, P. G. Bolhuis, and P. R. ten Wolde, Combining Molecular Dynamics with Mesoscopic Green's Function Reaction Dynamics Simulations, J. Chem. Phys. 143, 214102 (2015).

[26] A. Vijaykumar, T. E. Ouldridge, P. R. Ten Wolde, and P. G. Bolhuis, Multiscale Simulations of Anisotropic Particles Combining Molecular Dynamics and Green's Function Reaction Dynamics, J. Chem. Phys. 146, 114106 (2017).

[27] L. Sbailò and F. Noé, An Efficient Multi-Scale Green's Function Reaction Dynamics Scheme, J. Chem. Phys. 147, 184106 (2017).

[28] T. R. Sokolowski, J. Paijmans, L. Bossen, T. Miedema, M. Wehrens, N. B. Becker, K. Kaizu, K. Takahashi, M. Dogterom, and P. R. ten Wolde, eGFRD in All Dimensions, J. Chem. Phys. 150, 054108 (2019).

[29] В. Ø. Palsson, Systems Biology: Properties of Reconstructed Networks (Cambridge University Press, Cambridge, England, 2006).

[30] See Supplemental Material at http://link.aps.org/ supplemental/10.1103/PhysRevX.10.041032 for three additional figures provided.

[31] M. Doi, Theory of Diffusion-Controlled Reaction between Non-Simple Molecules. I, Chem. Phys. 11, 107 (1975).

[32] M. Doi, Theory of Diffusion-Controlled Reaction between Non-Simple Molecules. II, Chem. Phys. 11, 115 (1975). 
[33] S. Sarkar and A. E. García, Presence or Absence of Ras Dimerization Shows Distinct Kinetic Signature in Ras-Raf Interaction, Biophys. J. 118, 1799 (2020).

[34] V. A. Ngo, S. Sarkar, C. Neale, and A. E. Garcia, How Anionic Lipids Affect Spatiotemporal Properties of KRAS4B on Model Membranes, J. Phys. Chem. B 124, 5434 (2020).

[35] S. von Bülow, M. Siggel, M. Linke, and G. Hummer, Dynamic Cluster Formation Determines Viscosity and Diffusion in Dense Protein Solutions, Proc. Natl. Acad. Sci. U.S.A. 116, 9843 (2019).

[36] E. M. Ozbudak, M. Thattai, I. Kurtser, A. D. Grossman, and A. Van Oudenaarden, Regulation of Noise in the Expression of a Single Gene, Nat. Genet. 31, 69 (2002).

[37] M. Thattai and A. Van Oudenaarden, Intrinsic Noise in Gene Regulatory Networks, Proc. Natl. Acad. Sci. U.S.A. 98, 8614 (2001).

[38] A. Sanchez, S. Choubey, and J. Kondev, Regulation of Noise in Gene Expression, Annu. Rev. Biophys. 42, 469 (2013).

[39] R. Grima, Noise-Induced Breakdown of the MichaelisMenten Equation in Steady-State Conditions, Phys. Rev. Lett. 102, 218103 (2009).

[40] R. Grima, Intrinsic Biochemical Noise in Crowded Intracellular Conditions, J. Chem. Phys. 132, 185102 (2010).

[41] K. Mayawala, D. G. Vlachos, and J. S. Edwards, Spatial Modeling of Dimerization Reaction Dynamics in the Plasma Membrane: Monte Carlo vs. Continuum Differential Equations, Biophys. Chem. 121, 194 (2006).

[42] W. Y. C. Huang, S. Alvarez, Y. Kondo, Y. K. Lee, J. K. Chung, H. Y. M. Lam, K. H. Biswas, J. Kuriyan, and J. T. Groves, A Molecular Assembly Phase Transition and Kinetic Proofreading Modulate Ras Activation by SOS, Science 363, 1098 (2019).

[43] A. Klosin, F. Oltsch, T. Harmon, A. Honigmann, F. Jülicher, A. Hyman, and C. Zechner, Phase Separation Provides a Mechanism to Reduce Noise in Cells, Science 367, 464 (2020).

[44] C. M. Giver and B. Chakraborty, Effects of Intrinsic Fluctuations in a Prototypical Chemical Oscillator: Metastability and Switching, arXiv:1303.3048.
[45] D. Goldstein, M. Giver, and B. Chakraborty, Synchronization Patterns in Geometrically Frustrated Turing Rings, Chaos 25, 123109 (2015).

[46] A. J. McKane, T. Biancalani, and T. Rogers, Stochastic Pattern Formation and Spontaneous Polarisation: The Linear Noise Approximation and Beyond, Bull. Math. Biol. 76, 895 (2014).

[47] A. Duncan, S. Liao, T. Vejchodský, R. Erban, and R. Grima, Noise-Induced Multistability in Chemical Systems: Discrete versus Continuum Modeling, Phys. Rev. E 91, 042111 (2015).

[48] T. Biancalani, F. Jafarpour, and N. Goldenfeld, Giant Amplification of Noise in Fluctuation-Induced Pattern Formation, Phys. Rev. Lett. 118, 018101 (2017).

[49] D. Curtis, Master's thesis, California State Polytechnic University, 2017.

[50] S. Pednekar, J. Chun, and J. F. Morris, Simulation of Shear Thickening in Attractive Colloidal Suspensions, Soft Matter 13, 1773 (2017).

[51] O. Brandman and T. Meyer, Feedback Loops Shape Cellular Signals in Space and Time, Science 322, 390 (2008).

[52] A. Yan, G. Xu, and Z.-B. Yang, Calcium Participates in Feedback Regulation of the Oscillating ROP1 Rho GTPase in Pollen Tubes, Proc. Natl. Acad. Sci. U.S.A. 106, 22002 (2009).

[53] R. N. Gutenkunst, J. J. Waterfall, F. P. Casey, K. S. Brown, C. R. Myers, and J. P. Sethna, Universally Sloppy Parameter Sensitivities in Systems Biology, PLoS Comput. Biol. 3, e189 (2007).

[54] J. D. Weeks, D. Chandler, and H. C. Andersen, Role of Repulsive Forces in Determining the Equilibrium Structure of Simple Liquids, J. Chem. Phys. 54, 5237 (1971).

[55] S. H. Strogatz, Nonlinear Dynamics and Chaos with Student Solutions Manual: With Applications to Physics, Biology, Chemistry, and Engineering (CRC Press, Boca Raton, FL, 2018). 